\title{
La fabrique participative des politiques publiques sur la qualité de l'air au prisme d'un dispositif innovant : artivistes-atelier
}

\section{An innovative device using participatory decision-making to} inform public policy on air quality

\section{Davia Dosias-Perla', Lionel Scotto d'Apollonia², Sylvie Blangy ${ }^{3}$}

\author{
${ }^{1}$ Ingénieure CNRS, UMR ART-Dev (8251), membre GDR PARCS, davia.dosias-perla@cnrs.fr \\ ${ }^{2}$ Chercheur associé au LIRDEF (Université Montpellier), directeur adjoint GDR PARCS \\ lionel.scotto@fde.univ-montp2.fr \\ ${ }^{3}$ Ingénieure de recherche CEFE, CNRS, UMR (5175), Directrice GDR PARCS, sylvie.blangy@cefe.cnrs.fr
}

RÉSUMÉ. Cet article présente les premiers résultats d'une recherche s'appuyant sur un dispositif original Artivistes-atelier en recherche action participative analysant la fabrique participative des politiques publiques prenant en compte l'air, notamment les Plans Climat Air Énergie Territoriaux PCAET sur le territoire autour de Montpellier. En détaillant le design élaboré, l'article décrit le processus participatif permettant la construction d'une cartographie dynamique des actants impliqués/impactés et leur capacité d'action sur la qualité de l'air à l'échelle du territoire de Montpellier. Ce résultat permet de questionner par le prisme du dispositif Artivistes-atelier, les ressors et les potentialités de la mobilisation citoyenne relative à la démocratisation des enjeux socioenvironnementaux englués dans le mille institutionnel des modes de gouvernance territoriaux. Pour cela l'article décrit plus largement l'expérience de terrain dont est issu le dispositif, une expérience pragmatique, réflexive et critique. Cet article est l'occasion de construire un premier jalon comparatif s'intégrant dans un projet ADEME en R\&D, intitulé Air Climat Santé Société \& Art visant dans une logique de Recherche Action Participative à accompagner la mise en œuvre des politiques publiques en faveur de la qualité de l'air extérieur. Les résultats présentés dans l'article et les limites identifiées du dispositif Artivistes-atelier permettent de nourrir la réflexion sur les potentialités de prise en charge des enjeux sur la qualité de l'air extérieur par l'ensemble des acteurs d'un territoire avec comme tête de proue le citoyen dont la participation demeure à l'heure actuelle une arlésienne.

ABSTRACT. This article presents the initial results of a study on an original participatory action research approach focusing on the articulation and the participative fabric of public policies on outdoor air quality in Montpellier, France. The approach, called Artivistes-atelier, is described, providing a dynamic map of the stakeholders involved and/or impacted and their capacity for action. The article examines this field experiment that is simultaneously reflective and analytical. These initial results allowed to interrogate through the Artivistes-atelier initiative prism the strengths and potentialities of citizen mobilization and the socioenvironlental issues democratization which is ensnared in the institutional governance. For this purpose, the article describes more widely the experimental territory with a pragmatic, reflexive and critical experience. This article is a real opportunity to build a first comparative device to be reproduce in an ADEME project in R\&D, entitled Air Climate Society and Art. This project has two main aims: to decompartmentalize socio-environmental issues and to support the implementation of public policy on outdoor air quality. The results and identified limits of the Artivistes atelier device allow to examine the potentialities of the implementation of the public policies on air by all the stakeholders, starting with the citizen, whose participation in politics is an elusive phenomenon.

MOTS-CLÉS. Mobilisation citoyenne, gouvernance climatique, recherche action participative, Street art, accompagnement politiques publiques, sciences-sociétés, outil d'aide à la décision.

KEYWORDS. Citizen engagement, climate governance, participatory action research, street art, political action, influencing public policy, science and society, decision-making tools. 
«Les élus se sont tournés vers le dialogue avec les parties prenantes afin de contourner l'obstacle que constitue la mobilisation d'une minorité active, hostile aux projets avancés (...). Dès lors, l'exercice pratiqué ne constitue pas une volonté de modifier les pratiques démocratiques, mais représente une tentative visant à forger de nouveaux outils tactiques plus efficaces en phase de prise de décision. [...] Pensaient-ils vraiment qu'en initiant un processus de concertation, chacun allait s'exprimer sereinement et que le climat s'apaiserait aussitôt entre acteurs défendant des positions radicalement opposées ? (Buclet, Salomon, Les processus participatifs transforment-ils les représentations des élus ruraux sur leur rôle et leur territoire? Espaces et sociétés 2007, p.187)

«Accord de Paris, objectif $2^{\circ} \mathrm{C} \ldots$... Au-delà des discours, des textes, l'Atelier Climatique Citoyen ${ }^{1}$ offre de belles perspectives d'adhésion du plus grand nombre dans notre pays, dans nos territoires. Sensibilisation, concertation en sont des approches incontournables tout autant que l'art et culture en seront aussi de formidables vecteurs » (Jouzel, Jean. L'Atelier Climatique Citoyen, 2016)

\section{Introduction}

\subsection{Le contexte: un double déficit de mobilisation et d'efficacité des politiques publiques}

L'appel du « tout participatif » résonne actuellement comme la solution pour redonner du sens et de l'efficacité à notre système démocratique englué dans une crise profonde et empreint de tensions multiples. De nombreux travaux sur la participation citoyenne ont mis au jour l'abîme entre les ambitions affichées et le constat frappant d'une inefficacité chronique sur le terrain, la démocratie participative perdant peu à peu de sa substance [BEV 02] [BLO 08] [FOU 07] [BLO 11]. Bon gré, mal gré, les nouveaux modes de gouvernance dérivent vers des logiques gestionnaires [SAL 13], des cadres réglementaires aux échelles territoriales définissant les contours de la mise en place de changements sociaux, faisant office de laboratoire des expérimentations démocratiques. Sans doute émoussé par les critiques, le terme de gouvernance environnementale s'est transformé dans le champ lexical universitaire et politique en agir environnemental. Entre effet de cosmétique communicationnel ou réel glissement conceptuel, il ne s'agit plus d'investir les objectifs du développement durable mais de s'orienter dans la transition écologique, énergétique et solidaire $^{2}$ avec l'ambition de cadrer les objectifs de cette transition dans l'épaisseur de leur complexité, c'est-à-dire dans leur dimension environnementale, économique, sociale, juridique et politique. Pour caractériser l'intégration des «nouvelles» formes de la démocratie participative dans la démocratie représentative, il est commun de parler d'«innovation» habillée au gré des situations d'adjectifs aussi divers que variés. L'innovation (socioenvironnementale et urbaine), dans laquelle le dispositif Artivistes-

\footnotetext{
${ }^{1}$ L'Atelier Climatique Citoyen est le nom générique d'un dispositif participatif visant à décloisonner les enjeux climatiques et à accompagner les politiques publiques dans la mise en place de l'Accord de Paris 2015 sur les territoires.

${ }^{2}$ L'article reprend les termes du programme CIT'In co-piloté par le Ministère (bien nommé) de la transition écologique et solidaire et le monde de la recherche afin de porter un regard analytique sur des expérimentations démocratiques pour la transition écologique et énergétique. $C f$. site internet consulté le 3 mai 2018 sur le lien suivant: https://citin.hypotheses.org/.
} 
atelier s'inscrit et sur laquelle cet article s'appuie ${ }^{3}$, est perçue comme un enjeu majeur pour toute une série d'acteurs notamment ceux issus du monde, associatif, politique, de la recherche et de l'entreprenariat. Cependant, les efforts en matière d'innovation démocratique relatifs aux enjeux socioenvironnementaux se heurtent à une cohorte de freins plus ou moins bien identifiés. En effet, la société dite civile malgré les appels citoyens massifs reste peu mobilisée et représente un angle mort des politiques publiques dans leur mise en œuvre [COM 15 a]. Cette dépossession écologique ne touche pas uniquement les classes dites populaires [BOU 97] et irrigue l'ensemble de la société. Pour pallier ce déficit, les outils de la concertation numérique à travers les plates-formes de contributions en ligne, les «Civic tech»[MAB 17] apportent des résultats encourageants. Cependant, ils ne permettent pas de compenser pleinement ce déficit d'implication citoyenne ni de toucher des publics dont les habitus sont découplés des politiques environnementales. Dans le cas du climat ${ }^{4}$, les porteurs de causes ont tendance à dépolitiser le débat dans le but de construire une version consensuelle pour imposer une vision normative des «bonnes actions » à conduire, s'arrogeant par subsidiarité la possibilité de s'exprimer à la place de l'« éco-citoyen », catégorie fourre-tout masquant des rapports de lutte de classe [COM 15 b]. Or il est important de ne pas projeter des a priori sur les niveaux de sensibilisation ou de préoccupation des citoyens et sur leurs volontés d'agir mais bien au contraire de privilégier une attention à leurs expériences vécues, aux micro-mondes. [CHAT 17].

Force est de constater qu'il existe un double déficit : d'une part un déficit d'implication citoyenne sur les questions environnementales et d'autre part un déficit de mise en œuvre de politiques ayant des effets concrets [SCO 17]. Cet article présente le dispositif Artivistesatelier $^{5}$ conçu pour répondre à ce double déficit et les premiers résultats d'un atelier participatif conduit dans le cadre d'une phase préalable à la mise en route d'un projet de recherche et développement (R\&D) Air Climat Santé Société \& Art porté conjointement par l'association du même nom Artivistes-atelier ${ }^{6}$ et l'UMR ART-Dev ${ }^{7}$. Ce projet pilote lauréat

${ }^{3}$ En ce sens, le dispositif a été lauréat du prix de l'innovation périurbaine Direction Départementale des Territoires et de la Mer de l'Hérault.

${ }^{4}$ Le cas du climat est en ce sens emblématique et peine encore à concrètement se démocratiser. En effet, après l'Accord Paris-Climat 2015 et la vague d'enthousiasme soulevée, le chantier est à peine ouvert et il reste à le mettre en application à la fois au niveau national et à l'échelle des territoires. Les nombreuses initiatives (collectif C40, Cities Climate Leadership Group, European Climate Foundation, etc.) tentent d'apporter des solutions à la mise en application de l'Accord sur les territoires. Cependant à y regarder de plus près, i.e. au-delà des effets de loupes médiatiques, la mise en cohérence de l'Accord avec les politiques publiques territoriales se heurte à de nombreux obstacles.

${ }^{5}$ Le terme Artivistes est en fait un acronyme (Association de Redynamisation des Territoires par l'InnoVation Sociale des Transitions EnvironnementaleS). Nous le retrouvons également dans le monde du spectacle vivant. Une recherche indexée par année sur le moteur de recherche Google.fr démontre que le terme apparait sur la toile avec notamment un mouvement artistique de l'avant-garde berlinoise d'un collectif dénommé « Kotti Shop ». Il est possible de noter que le terme «artivistes » apparaît dans la littérature scientifique en 2010 [BAU 10] et plus précisément à partir d'une étude dans le centre historique de Rio de Janeiro à l'aune d'initiatives tournées vers la valorisation de formes patrimoniales caractéristiques des espaces d'occupation illégale que sont les favelas. Ces mouvements relèvent à la fois d'institutions publiques et $\mathrm{d}$ '«artivistes», identifiés comme «des acteurs engagés dans l'exploration des nouvelles possibilités offertes par la médiatisation et la valorisation tant économique que politique de l'intervention artistique dans l'espace urbain ».

${ }^{6}$ Cf. site internet consulté le 3 mai 2018 sur le lien suivant http://artivistes.neowordpress.fr/.

${ }^{7}$ L'Unité Mixte de Recherche ART Dev s'inscrit prioritairement dans le champ des sciences humaines et sociales. L'UMR privilégie les approches interdisciplinaires et développe des recherches sur les enjeux de reconfigurations territoriales en donnant un fort volet de recherche sur l'analyse des reconfigurations sur la construction et l'appropriation par l'ensemble des acteurs d'un territoire. $C f$. site internet consulté le 3 mai 2018 sur le lien suivant http://art-dev.cnrs.fr/spip.php?rubrique86. 
d'un appel à projet ADEME AACT-AIR ${ }^{8}$ vise à construire avec un groupe multi acteurs des indicateurs d'efficacité des politiques publiques en faveur de la qualité de l'air sur le territoire exploratoire de l'Agglomération du Pays de l'Or (Hérault), en s'appuyant sur les outils de la Recherche Action Participative (RAP) développés par la communauté de pratique canadienne de Jacques Chevalier ${ }^{9}$ et relayés par le GDR PARCS ${ }^{10}$ (Participatory Action Research and Citizen Sciences) en France. Les indicateurs sont devenus des instruments de pouvoir qui peuvent agir comme une boussole ou tout autant comme un miroir déformant de légitimation des politiques publiques [ZIT 09]. La construction en présence de l'ensemble des acteurs (élus, collectivités, citoyens, décisionnaires, agences étatiques, associations) d'indicateurs d'efficacité d'un Plan Climat Air Énergie Territorial (PCAET) au moment de sa formulation est un moyen original de construire et de collecter des données de recherche visant à analyser dès ses prémisses, la fabrique participative des politiques publiques sur l'air.

\subsection{Le dispositif Artivistes-atelier en réponse à ce double déficit :}

Le dispositif Artivistes-atelier vise à apporter des solutions concertées dans le cadre de la mise en place de politiques publiques tout en développant un regard analytique. L'étude de cas analysée dans cet article présente la particularité de porter sur un territoire ayant des niveaux de conflictualités «normaux » qui permettent le dialogue des parties prenantes et donc la réalisation d'un diagnostic partagé. Dans le cas de conflictualités moins « douces », le dispositif est en mesure de s'adapter en ayant pour seul objectif alors la réalisation et la production d'un diagnostic partagé. Le dispositif a la spécificité d'être hybride dans le sens où il est co-piloté par le milieu associatif, des citoyens, des étudiants, des artistes, des professionnels de l'environnement et des chercheurs de différents champs disciplinaires ainsi que certains issus du groupe de Recherche GDR PARCS. Il vise à décloisonner les enjeux socioenvironnementaux à destination de l'ensemble des acteurs d'un territoire. En ce sens, il se situe donc au cœur des rapports sciences-sociétés, objets d'intérêts accrus ces dernières années par les mondes: de la recherche, de l'entreprise, de la politique et du secteur associatif.

Le dispositif Artivistes-atelier investit tout un ensemble de problématiques comme celles de la biodiversité, de la gestion de la ressource en eau, de la qualité de l'air extérieur, enjeu traité dans le cadre de cet article, à différentes échelles territoriales (communes ou agglomérations ${ }^{11}$ ), avec comme point nodal le climat. La spécificité et l'originalité du dispositif consiste dans sa phase initiale à mobiliser le public le plus large possible ou à accompagner des mobilisations citoyennes existantes. Pour ce faire, il s'agit d'investir une zone cible, un quartier, un village, une ville ou un regroupement de communes au rang de $\mathrm{EPCI}^{12}$, en créant une rencontre avec les acteurs du territoire et les établissements scolaires

${ }^{8}$ AACT - AIR est le projet que nous avons soumis dans le cadre de l'appel à projet national porté par l'Agence nationale du Développement et de la Maîtrise de l'Energie (ADEME) et signifie : aide à l'action des collectivités territoriales et locales en faveur de l'air.

${ }^{9}$ Animée par Jacques Chevalier et s'appuyant sur ses travaux. Pour plus d'informations se référer au site internet consulté le 3 mai 2018 sur le lien suivant www.sas2.net.

${ }^{10}$ Le Groupe de Recherche labélisé par le CNRS. PARCS pour Participatory Action Research and Citizen Sciences. Cf. site internet consulté le 3 mai 2018 sur le lien suivant : www.gdrparcs.fr.

${ }^{11}$ Nous renvoyons pour plus de détails sur les projets d'accompagnement des politiques publiques environnementales sur les territoires au site internet consulté le 3 mai 2018 sur le lien suivant http://artivistes.neowordpress.fr.

${ }^{12}$ Les EPCI sont des Établissements Publics de Coopération Intercommunale. Depuis l'adoption de la réforme territoriale de 2010 et de la loi du 27 janvier 2014 de modernisation de l'action publique territoriale et d'affirmation des métropoles, sont des EPCI (art. L5210-1-1 du code général des collectivités territoriales- CGCT) : les syndicats de 
afin de construire collégialement une manifestation d'art de rue. Cette opération culturelle et citoyenne peut prendre de multiples formes: Street Art, danse, slam / poésie, thêâtre, marionnettes géantes, groupe de musique, exposition photographique; toujours dans l'objectif d'interpeler le citoyen dans son espace de vie quotidien. Le travail auprès des acteurs en amont et plus particulièrement auprès de l'ensemble du tissu associatif et des scolaires est une phase préparatoire essentielle. En effet, cette prospection correspond à un travail micellaire permettant de potentialiser la mobilisation et de dynamiser le territoire. Il vise ainsi à accroitre la mobilisation citoyenne à un plus large public et la possibilité de s'engager dans des groupes multi-acteurs qui pérennisent la mobilisation. En ce sens, l'adage de l'association Artivistes-atelier est de (re)donner la parole au citoyen et les moyens d'agir. Ces groupes multi acteurs peuvent avoir plusieurs déclinaisons et apporter différents résultats concrets et opérationnels : la construction de panel pour la concertation, la construction d'outils d'aide et d'accompagnement des politiques publiques et enfin, la construction d'indicateurs d'efficacité des politiques publiques sur les enjeux environnementaux. Les objectifs que le dispositif vise à atteindre grâce à des projets en cours ou par le montage de nouveaux projets, sont de permettre au citoyen d'être acteur à l'échelle de son territoire sur des problématiques locales qui le concernent directement et d'analyser son appétence à être acteur et les freins et les leviers relatifs à son intégration dans le processus de construction de la politique. Sur le plan politique, il vise plus généralement à relever le défi climatique [JOU 14] s'avérant particulièrement complexe pour les pouvoirs publics. En effet, l'esprit de l'Accord Paris encourage les politiques publiques à agir avec la mobilisation de ladite « société civile».

En retour, chaque action mise en cuvre par le dispositif sur le terrain agit comme un marqueur à la fois réflexif et critique ${ }^{13}$, de la fabrique participative des politiques publiques et permet ainsi d'identifier certains des freins et des leviers à l'implémentation de ces politiques publiques. En questionnant les possibilités de montée en généralité [BOL 91] [THE 06] le dispositif permet de questionner les évolutions des modes de gouvernance démocratique sur les questions socioenvironnementales. Le dispositif agit comme un kaléidoscope par sa capacité à générer de l'interaction entre les acteurs et ce à différents niveaux d'échelles territoriales et nationales, en regard du mille-feuille institutionnel. Le dispositif, aussi vertueux soit-il, soulève néanmoins des questions pouvant s'avérer problématiques du point de vue de la posture des chercheurs impliqués et du dispositif par rapport à la place du citoyen. En tant que dispositif critique et réflexif, il agit comme un artefact, véritable instrument analytique des expérimentations démocratiques. Ce travail est donc expérimental, son efficacité se mesurant à l'aune des résultats qu'il produira. Le pari est véritablement osé, cependant les hauts niveaux d'incertitudes peuvent s'avérer heuristiquement très féconds sur le plan de la recherche y compris en cas de crash de l'expérimentation.

communes; les communautés de communes; les communautés urbaines ; les communautés d'agglomération; les syndicats d'agglomération nouvelle et les métropoles.

${ }^{13}$ Il ne s'agit pas d'ouvrir ici les débats en cours sur la place de la critique dans le pragmatisme. Nous renvoyons le lecteur à ce propos vers les travaux du GSPR dirigé par Francis Chateauraynaud ( $C f$. site internet consulté le 3 mai 2018 sur le lien suivant http://www.gspr-ehess.com/ ). Une approche autant réflexive que critique vise à objectiver la prépondérance des contraintes normatives, juridiques et techniques. Il apparait hautement nécessaire de s'émanciper des logiques et des contraintes communicationnelles afin de questionner en profondeur les freins propres au labyrinthe interne de l'institution, aux agendas politiques et aux contraintes bureaucratiques apparaissant comme autant de facteurs limitants pour la traduction politique des accords (type Accord Paris Climat 2015) ou directives européennes (Directive Cadre sur l'Eau de 2010) etc. Il est donné en constat que la seule volonté des élus ne suffit pas à décloisonner la problématique pour rendre opérationnel et efficace ce projet national sur leur territoire. 
Le dispositif Artivistes-atelier a été créé par Davia Dosias-Perla, consultante en développement des territoires sur les enjeux socioenvironnementaux et animatrice territoriale dans le cadre de grandes concertations (Schéma d'Aménagement et de Gestion de l'Eau, Schémas Directeurs, Plans Climat ...); et par Lionel Scotto d'Apollonia, enseignant et chercheur spécialiste des enjeux climatiques. Artivistes-atelier, en partenariat avec l'UMR ART-Dev, a été lauréat en 2017, d'un projet ADEME AACT-AIR, dénommé Air, Climat, Santé, Société \& Art, dont l'article détaille les premiers résultats dans le développement d'une phase exploratoire. Tous deux ont constaté l'échec en termes d'appropriation des enjeux techniques environnementaux et politiques par les parties prenantes (élus, techniciens, décisionnaires privés et citoyens). L'expertise territoriale dont est issu le dispositif a permis en amont d'identifier et d'analyser les procédures administratives et réglementaires de construction de la politique publique sur l'air et leur articulation. En effet, cette construction malgré un effort de décentralisation reste essentiellement top down et prend racine dans une injonction au niveau étatique d'implémenter des actions concrètes et efficaces en termes de résultats ${ }^{14}$ sur les territoires. Deux exemples illustrent la décentralisation à marche forcée des enjeux sur l'air. D'une part, en 2016 l'enjeu « Air » a été délégué aux collectivités territoriales de façon brutale par l'introduction de la lettre «A» de «Air» dans le Plan Climat Air Énergie Territorial (PCAET). D'autre part, en mai 2018, la France était encore sous la menace d'un renvoi devant la Commission Européenne dans le cadre de deux procédures précontentieuses portant sur des dépassements de valeurs initiales en particules (PM10) et en dioxyde d'azote $\left(\mathrm{NO}_{2}\right)$. Elle n'avait toujours pas rendu à la Commission européenne un plan d'actions visant à réduire la pollution atmosphérique malgré un arrêté rendu en juillet 2017 par la plus haute juridiction, le Conseil d'État ${ }^{15}$. La réponse à cette injonction à agir se traduit sur les territoires ciblés, par la reformulation des Plans Prévention Atmosphère (PPA) dans l'urgence et dans le désordre avec les Préfectures et les DREAL ${ }^{16}$ en région en réunissant les parties prenantes qui ont les moyens et la volonté de se mobiliser dans l'urgence. Dans le PPA de l'aire urbaine de Montpellier ${ }^{17}$ par exemple le Comité de Pilotage se limite aux grosses collectivités, aux agences d'Etat (Agence Régionale de la Santé, ATMO Occitanie) et au secteur privé (Autoroutes Sud de France, $\mathrm{SNCF}^{18}$ ). Les citoyens sont absents de cette grande réunion de travail concertée ou seulement représentés en séance de clôture et de présentation de la feuille de route territoriale par de grosses associations nationales investies et engagées (France Nature Environnement). L'échec de la territorialisation de l'enjeu sur la qualité de l'air est en ce sens patent.

\footnotetext{
${ }^{14}$ Dont les critères et objectifs sont souvent renseignés dans les documents d'orientation des stratégies nationales.

15 Par décision du 12 juillet 2017, le Conseil d'État a enjoint le Gouvernement de mener des actions fortes pour l'amélioration de la qualité de l'air, afin d'enregistrer rapidement des progrès en matière de lutte contre la pollution atmosphérique.

${ }^{16}$ La DREAL, Direction Régionale Environnement Aménagement Logement est un service déconcentré de l'État rattaché au Ministère de la Transition Écologique et Solidaire et du Ministère de la Cohésion des Territoires. La DREAL est placée sous l'autorité du préfet de région.

${ }^{17}$ Un premier plan de protection de l'atmosphère (PPA) sur l'aire urbaine de Montpellier a été élaboré en 2006, puis révisé en 2014. Le PPA révisé (2014-2019), actuellement en phase active de mise en œuvre, propose un bouquet de 16 actions portant sur différents secteurs d'activité (transport, industrie, urbanisme, résidentiel et tertiaire, communication et plan d'urgence) et couvre 115 communes, représentant plus de la moitié de la population du département de l'Hérault. A l'heure actuelle, la zone de Montpellier est ciblée par les mesures de pré contentieux notamment sur les dépassements en $\mathrm{NO}_{2}$.
}

${ }^{18}$ SNCF, Service National de Chemins de Fer. 
Dans une logique pragmatique, le dispositif s'adapte à la rémanence des logiques top down et à la volonté affichée mais inefficace de vouloir pratiquer exclusivement du bottom up. En ce sens, le dispositif Artivistes-atelier questionne les différents niveaux d'échelles d'action, de gouvernance et de prise de décision politique et leurs interactions tout en gardant une logique d'une meilleure efficacité au sein des collectivités territoriales et locales. L'accompagnement de la mise en ouvre des politiques publiques en faveur de l'air passe par la compréhension et l'analyse du système d'organisation des institutions très sectorisées, des procédures administratives cloisonnées et d'un mode de gouvernance complexe des politiques publiques territoriales [DUB 09]. C'est un parti pris fort qui in fine vise à la mise en place des politiques publiques territoriales ${ }^{19}$ tout en restant capable de garder une attention aux citoyens et de capter éventuellement les mobilisations citoyennes spontanées. La dimension critique et réflexive du dispositif vise à lever au moins partiellement les tensions relatives à cette posture de recherche fortement normative. A ce stade rien ne permet de présupposer la réelle capacité du dispositif à mettre en synergie de façon opérante les «sphères » politiques et citoyennes, un hiatus durable s'étant en l'état actuel installé comme nous le fait remonter le terrain du projet Air Climat Santé Société \& Art dans le début de son lancement.

\subsection{Décloisonner l'enjeu sur l'air : co-construction d'une cartographie dynamique des acteurs sur la qualité de l'air}

Le dispositif Artivistes-atelier a été conçu de manière à pouvoir favoriser et analyser : (1) la mobilisation des acteurs ; (2) la dynamisation des territoires ; (3) l'accompagnement et la construction des politiques publiques. Comme l'article l'indique précédemment, ces trois entrées sont analysées à travers la construction d'indicateurs dans des groupes multi acteurs constitués dans le cadre du projet Air, Climat, Santé, Société \& Art. Les questions de recherche sont formulées sur la base des trois thématiques suivantes: Mobiliser/Dynamiser/Accompagner ${ }^{20}$ afin de permettre in fine d'identifier les freins et leviers de la mise en place des politiques engluées dans le mille-feuille institutionnel. Conscient et nourri par les difficultés, ce projet cherche en pleine conscience à se donner les moyens d'atteindre ses objectifs. Le système d'action visant à mettre en application les politiques publiques se heurte à la difficulté de définir de façon collective et concertée des indicateurs d'efficacité pertinents [MON 92]. En effet, ces indicateurs doivent prendre en compte les perspectives dynamiques et transformatives d'autres systèmes d'actions dont les effets mobilisent différentes échelles de temps et d'espaces, mais aussi être socialement acceptés afin de pérenniser ces actions.

\footnotetext{
${ }^{19}$ L'efficacité du dispositif repose en ce sens sur une volonté de développer une approche autant réflexive que critique visant à objectiver la prépondérance des contraintes normatives, juridiques et techniques permettant d'objectiver autant que faire se peut la subjectivité des logiques d'actions et de leur efficacité. Il apparait hautement nécessaire de s'émanciper des logiques et contraintes communicationnelles afin de questionner en profondeur les freins propres au labyrinthe interne de l'institution, aux agendas politiques et aux contraintes bureaucratiques apparaissant comme autant de facteurs limitants pour la traduction politique des accords (type Accord Paris Climat 2015) ou directives européennes (Directive Cadre sur l'Eau de 2010) etc. Il est donné en constat que la seule volonté des élus ne suffit pas à décloisonner la problématique pour rendre opérationnel et efficace ce projet national sur leur territoire.

${ }^{20}$ A titre d'exemple, voici quelques questions de recherche : (1) Quels sont les apports et les limites du dispositif Artivistes-atelier concernant la mobilisation et l'appropriation citoyenne dans la mise en application des politiques publiques climatiques sur un territoire ? (1 bis) Quels sont les indicateurs pertinents pour évaluer la mobilisation et l'appropriation citoyenne? (2) Quels sont les avantages et les limites du dispositif dans l'application et l'accompagnement des politiques climatiques ? (2 bis) Quels sont les indicateurs pertinents pour évaluer l'efficacité des actions conduites ? ( 2 ter) Comment la dynamique des groupes multi-acteurs agit-elle en retour sur les autres acteurs par boucles de rétroactions?
} 
Pour illustrer notre propos l'article puise dans les enseignements tirés de l'organisation d'un atelier participatif test conduit dans le cadre de la redéfinition du projet Air Climat Santé Société \& Art. Cet atelier participatif s'ancre sur quelques questions qui ont permis d'orienter et d'analyser les résultats de l'atelier. L'ADEME dans une première évaluation ayant retenu le projet déposé par Artivistes-atelier/ART-Dev (Mai - Juin 2017) a demandé d'affiner la proposition et de donner des précisions sur les modalités de mobilisation citoyenne. Les premières Assises Sciences-Sociétés ${ }^{21}$ ont été l'occasion d'animer cet atelier participatif qui visait à apporter des précisions au comité d'évaluation de l'ADEME. Le design de l'atelier a été réalisé en équipe avec l'appui des membres du GDR PARCS et a été pensé dans l'objectif de construire une cartographie actants/actions en apportant des réponses aux questions suivantes :

- Quelles sont les questions que vous vous posez sur l'air?

- Quels sont les problèmes auxquels vous êtes confrontés?

- Quelles sont selon vous les mesures à prendre?

- Comment faudrait-il faire pour suivre efficacement ces mesures?

Les résultats de l'atelier participatif prennent la forme d'une cartographie dynamique des actants (acteurs et institutions) et des facteurs de mise en œuvre des actions visant à identifier de façon pondérée leur degré d'engagement et leur capacité d'action dans les processus décisionnels sur l'enjeu de la qualité de l'air à l'échelle du territoire de la Métropole Montpelliéraine. Pour cela, l'article détaille la construction des outils spécifiques développés dans le cadre de l'atelier participatif sur la qualité de l'air et de manière transversale les enjeux climatiques associés. Ces outils ont comme objectif d'être reproductibles par la suite dans les municipalités du territoire d'étude (Pays de l'Or) sous la forme d'un dispositif stable. L'article présente également les détails méthodologiques qui ont permis la production des résultats de l'atelier participatif. L'entrée s'est effectuée par familles d'actions potentielles à appliquer sur le territoire, ainsi, par étapes successives, l'atelier a permis d'objectiver les catégories d'acteurs en fonction de leur pondération sur les différentes actions identifiées par les participants ${ }^{22}$.

\subsection{Plan de l'article}

Dans une première partie, l'article détaille les cadres de référence généraux mobilisés sur le plan théorique, dont est issu le dispositif Artivistes-atelier, la revue de littérature et présente les enjeux liés à la qualité de l'air dans leurs liens étroits avec les modes de vie et l'industrialisation.

La deuxième partie décrit les modalités opératoires du dispositif, à la fois sur le plan général, et sur la particularité liée à l'atelier participatif sur la qualité de l'air. Cette partie aborde également les aspects plus spécifiques notamment en lien avec la construction de la cartographie issue de l'atelier. Cette cartographie vise à identifier les acteurs clés les plus

\footnotetext{
${ }^{21}$ Les assises Sciences-Sociétés ont été organisées à Montpellier du 4 au 6 juillet 2017 par la MSH SUD, l'association Artivistes-atelier était partenaire et les trois auteurs de l'article étaient membres actifs du comité scientifique et d'organisation. $\quad C f$. site internet consulté le 3 mai 2018 sur le lien suivant : http://www.mshsud.org/valorisations/sciences-societes/assises-sciences-societes.

${ }^{22}$ Ces premiers résultats ont été présentés lors du $7^{\text {èe }}$ colloque de l'OPDE (Outils pour Décider Ensemble), qui s'est tenu en novembre 2017 à Montpellier. $C f$. site internet consulté le 3 mai 2018 sur le lien suivant : https://www.reseau- 
importants et ceux qui ont un intérêt à agir au sein de chaque action ou mesure politique en faveur de la qualité de l'air sur le territoire montpelliérain.

La troisième partie décrit les résultats de l'exercice intitulé «analyse des acteurs ». Il consiste à identifier les acteurs, qui ont le plus d'influence et de pouvoir décisionnel sur le projet et ceux qui, tout en étant affectés n'ont que peu de pouvoir décisionnel [CHEVI 09] [CHEVI 11]. Leur pouvoir d'action respectif permet de mettre en lumière les défis qu'il convient de relever pour modifier les comportements, renforcer les capacités et s'attaquer aux asymétries [MAY 05].

\section{Cadre théorique du dispositif}

De façon générale, le dispositif Artivistes-atelier est ancré dans la Recherche Action Participative développée par Jacques Chevalier et Daniel Buckles [CHEVA 07] et s'appuie sur un ensemble de champs théoriques que nous mobilisons au besoin de notre investigation et en fonction des logiques de notre terrain.

\subsection{Attentes citoyennes et représentations sociales}

Afin d'interroger les attentes citoyennes et plus largement les croyances et les théories socialement partagées sur les problématiques socioenvironnementales, sont mobilisés les travaux de Serge Moscovici [MOS 61], de Denise Jodelet [JOD 89] et ceux de Patrick Perretti-Watel et Béatrice Hammer [PER06] plus particulièrement sur les représentations profanes de l'effet de serre.

\subsection{Régulation politiques publiques}

Le dispositif général s'inscrit dans le cadre de l'évolution des rapports sciences-sociétés et des difficultés de régulation des décisions publiques en situation d'incertitude [CAL 09]. Les ateliers participatifs sont en ce sens considérés comme des espace ouverts ou forums hybrides dans lesquels des groupes d'acteurs débattent des choix techniques et de leurs modalités d'application. Les modalités de régulation des politiques publiques sont considérées à partir de la notion globale de gouvernance environnementale [BAR 11], caractérisant une plus grande ouverture dans le processus décisionnel à tout un ensemble d'acteurs [PIT 10]. La transformation des modes de gouvernement est considérée en tant que principe normatif d'action pour désigner des principes et des méthodes standards pour une «bonne gouvernance» des problèmes publics. De plus, elle est entendue comme rhétorique du débat public et de l'action publique [LE G 95] ainsi que rhétorique du développement territorial et environnemental [THE 02]. Bien que de nombreux travaux aient pointé les limites d'une approche globalisante thématisée sous le concept de modernité réflexive [BEC 01 [86]], l'approche du débat vis-à-vis des formes émergentes de démocraties participatives [BLO 08] [FOU 07] [BLO 11] s'inscrit dans les grandes lignes des schémas habermassiens [HAB 87 ; 97]. Les changements sociaux inhérents mettent en jeu de nombreuses catégories d'acteurs et inévitablement différents niveaux de conflictualités. Les études ont mis en évidence la capacité de ces groupes d'acteurs à se construire des compétences suffisantes pour influencer le débat public, mais aussi accroître une demande légitime d'informations sur des sujets soumis à de fortes incertitudes. Par ailleurs, les demandes des publics concernant ces questions sont bien plus complexes et respectables que ce que présentent les arènes médiatiques [CHEVI 03]. 


\subsection{Praxis réflexive}

Le modus operandi s'adapte de façon pragmatique et réflexive en permanence aux logiques du terrain [CEF 07] [CHAT 15]. La réflexivité est appréhendée sur l'ensemble des niveaux d'analyse, individuels, collectifs et institutionnels. Elle se base notamment sur les travaux de Pierre Bourdieu [BOU 01] et plus récemment de ceux de Joëlle Le Marec [LE M 10]. La réflexivité qui est au cœur de ce projet de recherche doit être appréhendée en considérant les tensions entre deux niveaux en interaction : le niveau individuel par la mobilisation de la propre normativité des acteurs et le niveau des institutions. Le projet Air Climat Santé Société et Art s'inscrit dans différents champs de la recherche ayant abordé des thèmes comme celui de l'air, du climat, et plus généralement celui de l'environnement en intégrant notamment le champ émergeant de la sociologie de l'énergie [ZEL 14].

\subsection{Dimension participative}

La multiplication des dispositifs de concertation et de participation s'est imposée dans le monde de l'expertise, de l'innovation technique et sociale et de la recherche, répondant à des attentes, potentiellement paradoxales, de plus de démocratie et en même temps d'une meilleure efficacité en termes de gestion environnementale et sociale [MER 05] [SAL 06].

Notre projet de recherche, à travers le dispositif Artivistes-atelier s'équipe de procédures et d'outils collaboratifs que nous considérons comme nécessaires pour fluidifier les échanges et corriger les asymétries entre acteurs, pour garantir une participation plus équilibrée de tous les acteurs et pour assurer autant que faire se peut une transparence de l'information, pour évaluer les résultats de l'action collective et pour exiger des acteurs qu'ils rendent des comptes sur les résultats de l'action collective [PEC 05]. La dimension participative que nous déployons avec l'appui de la Recherche Action Participative et ce avec une proximité affichée avec le GDR PARCS et Jacques Chevalier [CHEVA 09] représente une des composantes intrinsèques de la gouvernance environnementale. Les limites de l'engagement citoyen et la capacité des acteurs à s'insérer dans de réels programmes de sciences participatives [GON 14] tout en respectant les positions axiologiques de chaque acteur du projet sont des limites à identifier et analyser [BOE 12].

\subsection{Acteurs clés}

L'atelier participatif dont l'article présente les résultats a permis une identification des parties prenantes ayant un pouvoir d'action sur les politiques publiques locales concernant la qualité de l'air. Cette identification aide à choisir la méthode pour identifier les principaux intervenants ou institutions concernés par les enjeux de qualité de l'air sur le territoire montpelliérain. L'identification se fait par pondération et analyse du degré d'influence et d'impact sur une situation clé ou un enjeu identifié par un large groupe d'acteurs [MAY 05].

\subsection{Qualité de l'air}

Concernant la qualité de l'air extérieur, le projet s'appuie sur le plan socio-historique des pollutions atmosphériques et des différentes pollutions et nuisances [CHAR 15]. La question de l'air y occupe une place très particulière en raison des liens étroits entre l'industrie, les enjeux sanitaires et nos modes de vie. Il existe un déficit de connaissance persistant concernant les analyses de l'impact sanitaire en raison de la législation réglementant une 
politique de seuils et de normes économiquement acceptables au détriment des besoins sanitaires. L'émergence du principe ALARA (As Low As Reasonably Achievable « Aussi bas que raisonnablement possible ») dans la directive IPPC (Integrated Pollution Prevention and Control) de 1996 [IPP 96] illustre bien ces propos.

\section{Méthodologie}

\subsection{Aspects généraux du dispositif Artivistes-atelier :}

Cette partie présente la méthodologie générale afin de clarifier le modus operandi relatif au dispositif Artivistes-atelier. Cette méthodologie n'est pas mobilisée dans le cadre de cet article. La description se limite à présenter sa phase initiale faisant l'originalité du dispositif.

Dans sa phase initiale, le dispositif général Artivistes-atelier vise à interpeler et à mobiliser le plus grand nombre d'acteurs par l'organisation d'une manifestation artistiques de rue. En fonction du contexte et des mandats, le dispositif permet de créer un maximum d'interactions y compris et en premier lieu avec les artistes.

Le dispositif prévoit de récolter directement sur une œuvre créée de façon participative, des représentations, des idées, des souhaits, des revendications. D'autres outils, comme à titre d'exemple : une acuponcture urbaine, l'atelier mon oil, l'arbre à palabres climatiques ${ }^{23}$ sont mobilisés en fonction de la configuration et des objectifs de l'opération. Ces outils ont été créés à partir d'expertise de terrain en réponse soit à des mandats des collectivités, de la recherche action participative, de l'Etat ou en réponse à des initiatives. Ils ont été conçus dans une approche pragmatique et viennent nourrir l'analyse des données tout en permettant aux citoyens d'apporter leur regard sensible sur les sujets traités et d'intégrer le processus d'analyse des données.

Les données citoyennes récoltées à partir des dispositifs de rue constituent après une analyse partagée avec les acteurs, un premier diagnostic des besoins locaux et attentes citoyennes. Ces données sont croisées et comparées avec les diagnostics établis par les collectivités dans le cadre de grands projets environnementaux ou enquêtes publiques ainsi qu'avec les besoins des institutions (à travers des entretiens avec les élus et services techniques notamment). Le dispositif permet de questionner les limites de cette posture de Recherche Action Participative. Il peut intervenir en réponse à un mandat précis des collectivités, des entreprises, ou des citoyens eux-mêmes. Le groupe multi acteurs générera alors autour d'ateliers participatifs, des indicateurs d'efficacité et de faisabilité socioenvironnementale des actions de politiques publiques existantes ou en cours de reformulation.

\footnotetext{
${ }^{23}$ L'acuponcture urbaine est un terme que l'on retrouve dans les travaux d'urbanisme tactique du chercheur et de l'urbaniste Mike Lydon et Antony Garcia [LYD 15]. Mais aussi comme ingénierie de projet utilisée notamment par l'ancien maire de Curitiba au Brésil en prenant en compte les aspects sociaux de la ville, environnementaux à l'échelle des quartiers et économiques à l'échelle des familles et avec pour le citer "peu de moyens et saupoudré de créativité » [LYD 15]. L'arbre à Palabres climatiques est un outil de RAP créé par Lionel Scotto d'Apollonia ( $C f$. site internet consulté le 3 mai 2018 sur le lien suivant : https://parcs.hypotheses.org/larbre-a-palabres ). Enfin, l'atelier mon œil est un outil créé par Davia Dosias Perla permettant aux habitants et participants d'un quartier et d'une commune d'être acteur et de s'approprier l'événement en envoyant en instantané leurs visions des ateliers et de la manifestation qu'ils vivent sur leur territoire sous la forme de photos ou courtes vidéos à une adresse générique $(C f$. site internet consulté le 3 mai 2018 sur le lien suivant : http://artivistes.neowordpress.fr).
} 
Le dispositif Artivistes-atelier prévoit une évaluation intégrée à sa démarche pour assurer ainsi un retour réflexif et interroger la portée même du dispositif.

Les différentes phases se déroulent en 3 étapes :

(1) Phase préparatoire: [Objectifs : (a) générer une dynamique citoyenne et (b) établir un pré-diagnostique des besoins locaux].

(2) Phase évènement: [Objectifs : (c) cartographier les attentes citoyennes, (d) générer un groupe de pilotage multi-acteurs et (e) formaliser l'ébauche d'une feuille de route d'actions].

(3) Phase opérationnalisation : [Objectifs : (f) Conduire un groupe de pilotage qui (g) analyse la faisabilité et l'efficacité sociale et environnementale des actions proposées, (h) construire des indicateurs d'efficacité et un guide des actions à entreprendre ou des politiques publiques à mener].

\subsection{Contexte, objectifs et méthodologie de l'atelier participatif sur la qualité de l'air}

L'atelier s'est déroulé dans une des cours du site St Charles de l'Université Paul Valéry, Montpellier 3, le 5 juillet 2017, à 14h dans le cadre des assises Sciences-Sociétés. L'atelier a été le lieu d'incubation et de réorientation du projet Air Climat Santé Société et Art qui a démarré officiellement en décembre 2017.

L'objectif de cet atelier participatif était d'élaborer un design d'atelier exploratoire afin : (1) de le tester, (2) de construire des données permettant d'affiner le projet soumis à l'ADEME. Dans le cadre spécifique de l'appel à projet de l'ADEME, quelques précisions nous ont été demandées. Nous avons profité de cet exercice pour présenter un test méthodologique sur l'enjeu spécifique des politiques publiques sur la qualité de l'air. En ce sens, l'objectif de l'atelier était double :

1) cartographier les acteurs et parties prenantes d'actions qui favoriseraient la qualité de l'air sur le territoire de la Métropole de Montpellier

2) analyser le pouvoir décisionnel et les degrés d'influence de ces acteurs sur la réalisation et l'efficacité des actions identifiées.

Notons, qu'à ce stade de développement du projet Air Climat Santé Société \& Art (phase de démarrage) il n'a pas encore été possible, mais il est prévu de tester l'efficacité et la nature des indicateurs de mobilisation citoyenne sur cet enjeu. En effet il est important de souligner que le contexte des Assises Sciences-Sociétés n'a pas permis de constituer un groupe d'acteurs hétérogènes, la plupart des participants étant déjà sensibilisés aux enjeux d'air et de climat. Une analyse rapide de la sociologie des Assises permet de constater qu'il est difficile de mobiliser au-delà du cercle étroit des personnes investies dans ce type de cause et le giron associatif présentant des liens étroits avec la recherche. Ce type de remarque s'adresse tout autant à nous-mêmes. Aussi faut-il questionner la posture visant à légitimer la parole citoyenne sans toutefois dans le cas présent parvenir à la capter véritablement.

Malgré un effort de construire, la veille de notre atelier, un «Hors les murs » attractif, artistique et créant des espaces de dialogues conviviaux avec l'association Artivistesatelier $^{24}$ et un appel aux relais médias important avec un impact quantitatif fort (La

${ }^{24}$ Les détails et outils créés pour l'événement $C f$. site internet consulté le 3 mai 2018 sur le lien suivant : http://artivistes.neowordpress.fr/evenement-hors-les-murs-assises-sciences-societes/. Dans le cadre du «Hors les Murs » un autre atelier participatif a été conduit en extérieur en partenariat avec le laboratoire LIRMM le débat numérique. Ce 
Marseillaise, La Gazette Montpellier, les journaux quotidiens locaux, la Gazette en ligne, Radio Aviva, Radio Campus, France Bleu Hérault), les limites inhérentes aux calendriers (début des vacance scolaires et fin d'année universitaire), aux moyens déployés pour décloisonner les espaces institutionnels et informels n'ont pas permis une mobilisation citoyenne importante et diversifiée lors de la manifestation.

\subsection{La collecte et l'analyse des données}

Plusieurs instruments ont été utilisés pour la collecte des données. Certains visaient à analyser les représentations des personnes mobilisées sur la qualité de l'air, tandis que d'autres servaient à mobiliser leurs idées et leur représentation quant aux mesures et actions potentielles à mettre en place sur le territoire pour agir en faveur de la qualité de l'air. Les résultats présentés ici sont issus de trois instruments de collecte de données et d'analyse (chaises musicales, familles d'actions, cartographie des acteurs) issus du guide de Jacques Chevalier [CHEVA 11]. Ils portent spécifiquement sur les perceptions de la relation d'influence des acteurs impliqués ou ceux qu'il serait avantageux et nécessaire d'impliquer dans la prise de décision politique portant sur l'enjeu de la qualité de l'air.

L'atelier a été filmé, enregistré, pris en photo et retranscrit. Il a fait l'objet d'une analyse collaborative de contenu avec les participants et d'un compte rendu remis aux participants $a$ posteriori.

\subsection{Design de l'atelier participatif sur la qualité de l'air}

De manière générale, les esquisses du design des ateliers participatifs que nous avons conçues en équipe pour cet atelier font aussi appel au guide de la recherche action [CHEVA $11]^{25}$. Les esquisses du design visaient initialement à construire : (1) une cartographie des acteurs, (2) une cartographie des représentations (et des attentes), (3) une cartographie des actions, (4) l'identification de la sociologie du groupe multi-acteurs pouvant être créé, (5) la construction des indicateurs, (6) les freins et les leviers de la mise en œuvre des actions et la construction d'outils d'aide à la décision. Le design final s'est limité aux points précédents écrits en bleu, à savoir les points (1), (2) en partie, (3), (4) et (6).

La phase de préparation (dont le design et l'agenda de l'atelier) est essentielle pour la réussite de l'atelier. Elle se fait un groupe et consiste à remettre l'ouvrage sur le métier plusieurs fois en tenant compte de l'évolution des questions et des attentes des organisateurs. L'élucidation de la ou des questions de recherche étant la phase la plus délicate. Les chercheurs/animateurs ont tendance à vouloir couvrir trop de questions pour un temps de travail limité [BLA 17].

Nous avons fait ici le choix d'entrer dans les données par l'identification des parties prenantes [CHEVA 11]. Cette technique permet aussi bien de rendre compte des différences entre les acteurs clés d'un enjeu environnemental donné sur un territoire précis (ici, la qualité de l'air à l'échelle du territoire de la Métropole de Montpellier). Certaines parties prenantes peuvent être touchées par un plan d'action en faveur de l'air, et d'autres peuvent avoir une influence sur son développement.

dernier est visible sur la vidéo en lien sur le site internet consulté le 3 mai 2018 sur le lien suivant: http://artivistes.neowordpress.fr/assises-sciences-societes/.

${ }^{25}$ Les techniques sont issues du guide de la recherche-action, la planification et l'évaluation participatives $C f$. site internet consulté le 3 mai 2018 sur le lien suivant : www.sas2.net. 


\section{Phase initiale}

L'enjeu clé de l'atelier a été déterminé par le contexte du projet Air Climat Santé Société \& Art. Les questions ont été choisies en vue d'affiner la rédaction du dit projet dont l'objectif est d'aider les collectivités à la mise en œuvre des politiques publiques en faveur de l'air sur leur territoire.

\section{Phase de démarrage}

La phase de démarrage donne le ton. L'objectif de l'exercice aux participants et la ou les méthodes qui vont permettre d'atteindre collégialement cet objectif sont proposées aux participants.

En début d'atelier, l'animateur et son co-animateur inscrivent sur un tableau de papier blanc les grands axes de questionnements pour démarrer le travail participatif de construction d'une cartographie des acteurs et actions pour une politique publique en faveur de la qualité de l'air plus efficace :

a. Quelles sont les questions que vous vous posez sur l'air?

b. Quels sont les problèmes auxquels vous êtes confrontés?

c. Quelles sont selon vous les mesures à prendre?

d. Comment faudrait-il faire pour suivre efficacement ces mesures?

Ces questions ont servi de trame de discussion tout au long de l'atelier et ont été abordées indirectement au fil des exercices : brise-glace, échanges informels, familles de mesures, debriefing en fin d'atelier. Dans l'objectif d'une analyse comparative des méthodes et des résultats de cet atelier, répondre formellement à ces questions n'a de sens que si le travail d'atelier participatif est reproduit plusieurs fois sur le même territoire. Or le projet Air Climat Santé Société \& Art au moment de l'atelier participatif sur l'air, n'avait pas encore arrêté son territoire d'exploration.

\section{Déroulé de l'atelier}

L'atelier a duré une heure quarante-cinq et a été découpé en quatre phases afin de tester trois instruments de Recherche Action Participative et d'analyse sociale: les chaises musicales dont l'objectif est de recueillir les représentations des participants autour d'une situation donnée et d'obtenir une tendance des représentations plurielles; les familles flottantes d'action qui ont l'intérêt de regrouper les participants en familles d'intérêts autour d'actions qu'ils auront auparavant identifiées comme importantes à développer et enfin, l'identification et l'analyse des parties prenantes qui permet d'obtenir une cartographie dynamique et pondérée des parties prenantes et des actions identifiées dans le but de permettre a posteriori une analyse de leur degré d'impact sur l'action ciblée par les participants [CHEVA 11].

«Étape $1:$ les chaises musicales »

Les participants sont invités à se positionner assis sur des chaises, en cercle. Un participant reste debout, au centre, sans chaise. Une question est posée par ce participant au milieu du cercle afin d'obtenir les représentations de chacun sur le problème clé à savoir la qualité de l'air : "Pour moi la qualité de l'air c'est important car : .... ». Les personnes assises et d'accord avec son affirmation se lèvent en signe d'assentiment. L'animateur et le preneur de notes recueillent les affirmations et les scores (nombre de personnes debout et d'accord). Cet exercice bien qu'il soit simple est riche d'enseignements. Il permet de recueillir les représentations de chacun qui permettent la construction du discours autour de phrases et d'affirmation sur la qualité de l'air ainsi que le positionnement dans l'espace des 
représentations de chacun [BOY 15]. Les résultats sont pondérés en fonction du nombre de personnes adhérant à l'affirmation. Tous les participants ne sont pas obligés de venir au centre et de proposer une affirmation.

«Étape 2 : les familles flottantes d'action»

Par la suite, les participants inscrivent sur des panneaux de cartons blancs ou des post-its des mots clés et verbes d'action autour de la question suivante : A l'échelle de la Métropole montpelliéraine, quelle action, quelle mesure vous semble pertinente à renforcer ou à créer pour agir en faveur de la qualité de l'air ? Une fois l'exercice d'écriture terminé, les participants circulent dans l'espace, se rencontrent, s'interpellent, se questionnent sur le sens des mots clés de chacun. Des conversations souvent animées s'en suivent et permettent de passer à la suite de l'exercice. Ils sont alors invités par l'animateur à se regrouper en «famille d'action » en cherchant les complémentarités ou similitudes entre leurs mots clés. Des participants peuvent se retrouver «orphelins », il leur est alors proposé de se faire adopter par une famille déjà constituée. Les familles existantes peuvent également prendre l'initiative d'intégrer de manière pertinente les «orphelins» dans leur action. Les « orphelins » peuvent se permettre de constituer dans la mesure du possible, une nouvelle famille d'action qui sera développée dans l'étape suivante de l'atelier.

«Étape 3 : identification des parties prenantes : diagramme arc en ciel »

Une fois les familles d'action constituées, les participants sont invités à se regrouper et à discuter de leur action commune et à la nommer en tenant compte des mots clés qui les ont réunis. Les participants débattent de la faisabilité de ces actions : quels sont les acteurs et parties prenantes intervenant dans la réalisation de cette action en faveur de la qualité de l'air? L'objectif de cette technique est d'identifier les parties prenantes impliquées dans une action concrète qui vise à améliorer la qualité de l'air. Les participants répondent alors à la question suivante : comment améliorer la qualité de l'air à l'échelle de la Métropole de Montpellier ? Les acteurs identifiés sont les acteurs, les parties prenantes les plus importantes, celles qui ont un intérêt dans la situation en cours d'évaluation [MAY 05]. Cette technique appelée "analyse des acteurs » permet de dresser une cartographie des acteurs, d'analyser les choix d'action ou de mesure que chaque participant a fait émerger.

Les données seront analysées pour et par les futurs groupes multi acteurs qui se constitueront dans les ateliers que nous organiserons sur le territoire du Pays de l'Or et qui viendront en accompagnement des politiques publiques sur la qualité de l'air dans le cadre du projet Air Climat Santé Société \& Art.

Chaque famille d'action écrit ou dessine le nom ou la catégorie sociale de chaque partie prenante sur une carte distincte. Si la liste est trop longe, les participants peuvent regrouper les cartes et les rassembler les parties prenantes similaires ou proches ${ }^{26}$. Les parties prenantes ont nécessairement une influence ou sont impactées par l'action.

L'équipe animatrice a tracé un diagramme en forme d'arc-en-ciel sur un tableau au mur qui est divisé en trois parties égales : une à la gauche, une au milieu et une à la droite (Figure 5). 
Dans le demi-cercle au plus près de la base (le plus petit) les participants placent les post its des parties prenantes et acteurs qui sont les plus touchées, qui ont le plus d'influence et/ou les deux par l'action qu'ils ont identifiée. Dans le demi-cercle du milieu, nous retrouvons les post its des parties prenantes et acteurs qui sont moyennement touchés, qui ont moyennement d'influence et / ou les deux, et dans le grand demi-cercle, les post its des parties prenantes et acteurs qui sont le moins touchés, qui ont le moins d'influence et/ou les deux. Voir l'exemple Figure 5.

De gauche à droite du diagramme, se dessinent les spécificités suivantes : acteurs et parties prenantes ayant une influence sur l'action, puis au centre ceux qui influencent et sont impactés par l'action, enfin, à droite les parties prenantes ou acteurs qui sont impactés par l'action. Les «tranches» du diagramme décrites ci-dessus sont ensuite pondérées en fonction du degré propre à chaque demi-cercle : le plus, moyennement, le moins. Nous avons fait le choix d'avoir des codes couleur (mauve, rose, vert) qui permettaient la lecture finale des pondérations des acteurs et parties prenantes associés à chaque action sur le diagramme.

Les participants placent alors les post its en fonction de cette pondération, qui se veut dynamique. Les participants peuvent reproduire les post-its et placer les acteurs et parties prenantes plusieurs fois quand ceux-ci se retrouvent dans des postures différentes simultanément. Le placement des acteurs et parties prenantes doit faire consensus au sein de chaque famille d'action ou de mesure. Un rapporteur, complice de l'animateur fait le tour des tables pour rendre compte de ces moments de débats. Un enregistreur est placé sur chaque table de famille d'action.

De façon complémentaire, la méthodologie envisage d' " externaliser» et de renforcer l'analyse par des méthodes classiques d'enquêtes. Il s'agit d'effectuer auprès des acteurs des entretiens ouverts afin de questionner leur représentation et leur attente vis-à-vis de problématiques comme par exemple celle alliant qualité de l'air, santé et climat.

\section{Résultats}

\subsection{Résultats de l'atelier participatif}

\subsubsection{Les participants}

Les quatorze participants de l'atelier étaient inscrits aux Assises Sciences-Sociétés et pour certains, étaient membres du réseau Artivistes-atelier. Trois participants étaient des chercheurs membre du Groupe de Recherche PARCS (en sciences humaines et sociales mais aussi en sciences dites dures). Six participants étaient des citoyens issus de la société dite civile. Le reste des participants étaient des professionnels de l'environnement et des associatifs engagés sur les enjeux de qualité de l'air dans les enjeux de l'atelier. 


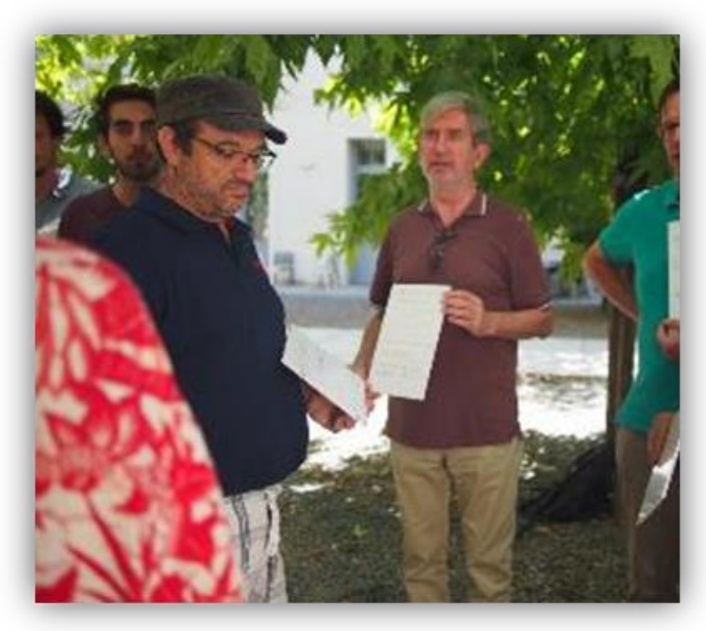

Figure 1. Elaboration des familles flottantes, Atelier qualité de l'air, Juillet 2017. C Sandra Barantal

\subsubsection{Résultats des chaises musicales}

$\mathrm{Au}$ cours de l'exercice «chaises musicales » 14 personnes sont allés à tour de rôle au centre du cercle et ont proposé une affirmation commençant par «Pour moi la qualité de l'air c'est important car... ». Les scores ou nombres de personnes d'accord avec les 12 affirmations sont élevés (de 14 à 12 personnes d'accord pour chaque affirmation sur 14 participants). Nous avons compilé les positionnements des participants détaillées dans le tableau 1.

\begin{tabular}{|c|c|}
\hline Pour moi la qualité de l'air c'est important car .... & Nbre debout/14 \\
\hline Pour bien respirer & $14 / 14$ \\
\hline C'est un bien universel & $14 / 14$ \\
\hline Elle renforce et cristallise les inégalités sociales & $14 / 14$ \\
\hline Ça participe à la longévité de la vie (rapport au taux de mortalité) & $13,5 / 14$ \\
\hline On a une meilleure espérance de vie et on reste en bonne santé & $13 / 14$ \\
\hline Je voudrai pouvoir circuler à vélo quotidiennement dans le futur sans masque & $13 / 14$ \\
\hline Pour la pratique sportive en ville & $12 / 14$ \\
\hline Il faut spatialiser car tout le monde n'est pas exposé de la même façon & $12 / 14$ \\
\hline Je ne veux pas payer de ma propre santé une pollution que je n'ai pas créée & $12 / 14$ \\
\hline Ça questionne les réaménagements des quartiers et des villes entières & $12 / 14$ \\
\hline $\begin{array}{c}\text { Elle est aussi importante que la qualité de l'eau mais l'eau, il n'y en pas } \\
\text { partout. Si l'air est pollué tout le monde l'est dans un référentiel donné. }\end{array}$ & $10 / 14$ \\
\hline \begin{tabular}{l} 
Ça développe beaucoup de moyens de recherche pour la voiture électrique \\
\hline
\end{tabular} & $10 / 14$ \\
\hline
\end{tabular}

Tableau 1. Résultats de l'exercice « chaises musicales » sur la qualité de l'air, Juillet 2017 


\subsubsection{Résultats des familles de mesures et actions en faveur de la qualité de l'air}

Trois familles se sont constituées après échanges et rencontres. Les résultats ont été retranscrits dans le tableau car les photographies des supports manuscrits n'étaient pas suffisamment lisibles.

Sur les panneaux des actions nous retrouvons les mots clés suivants : "pédagogie et sensibilisation », «transition et modification des modes de vie », " Aide à la mobilité verte et aux transports collectifs », « Développer des actions personnelles », ...

\begin{tabular}{|c|c|}
\hline $\begin{array}{l}\text { Groupes pour création de } \\
\text { famille d'action }\end{array}$ & Retranscription des mots clés des participants réunis en famille d'action \\
\hline Groupe 1 & $\begin{array}{ll}\text { - } & \text { Développer le vélo électrique (prêtés) et les pistes cyclables } \\
\text { - } & \text { Verdir les rues et fleurir les trottoirs } \\
\text { - } & \text { Expliquer que l'air est partagé entre les personnes et les moteurs } \\
\text { - } & \text { Multiplier les modes de déplacements doux } \\
\text { - } & \text { Développer les surfaces naturelles } \\
\text { - } & \text { Renforcer les transports collectifs } \\
\text { - } & \text { Remplacer les arbres abattus avec l'urbanisation } \\
\text { - } & \text { Développer la marchabilité en ville } \\
\text { - } & \text { Améliorer l'énergie dans l'habitat } \\
\text { - } & \text { Développer les parcs urbains }\end{array}$ \\
\hline Groupe 2 & $\begin{array}{ll}\text { - } & \text { Favoriser les transports en commun et la mobilité douce } \\
\text { - } & \text { Agir sur les activités qui génèrent des nuisances } \\
\text { - } & \text { Identifier les activités qui génèrent des nuisances } \\
\text { - } & \text { Préserver les espaces verts } \\
\text { - } & \text { Transports en commun } \\
\text { - } & \text { Mobilité douce } \\
\text { - } & \text { Chauffage / eau chaude solaire et thermique } \\
\text { - } & \text { Réussir à faire prendre conscience aux gens que chacun peut prendre des } \\
\text { - mesures à son échelle pour réussir à améliorer la qualité de l'air } \\
\text { - Comment concilier qualité de l'air et économie : exemple de l'aéroport } \\
\text { avec le tourisme }\end{array}$ \\
\hline Groupe 3 & $\begin{array}{ll}\text { - } & \text { Responsabiliser les citoyens via des objectifs personnels (compteurs } \mathrm{CO}_{2} \\
& \text { par foyer) } \\
\text { - } & \text { Rendre les transports en commun gratuit } \\
\text { - } & \text { Plus d'équité dans la gestion de l'air } \\
\text { - } & \text { Responsabiliser les citoyens } \\
\text { - } & \text { Développer des actions personnelles } \\
\text { - } & \text { Valoriser les actions individuelles }\end{array}$ \\
\hline
\end{tabular}

Tableau 2. Résultats de l'exercice "familles d'actions et de mesures » sur la qualité de l'air, Montpellier, Juillet 2017, Davia DOSIAS PERLA

\subsubsection{Résultats de l'identification des actants (acteurs et institutions) et parties prenantes par famille d'action}

Les participants se sont réunis par affinité de mots clés tel que présenté dans le tableau $\mathrm{n}^{\circ} 2$ et ont déterminé à quelle action en faveur de la qualité de l'air pouvaient correspondre leurs mots clés. Ils ont alors attribué un nom à leur famille représentant une action développée en faveur de la qualité de l'air dans le cadre de l'aide à la mise en place des politiques publiques territoriales.

Sont ressortis trois familles d'action : 
(1) Groupe 1 (post its vert) : Monsieur et Madame Velogratos à l'ombre ont deux enfants : Communiquer et Eduquer,

(2) Groupe 2 (post its rose) : Transition énergétique et aménagement de l'espace,

(3) Groupe 3 (post its mauve) : Donner un réel pouvoir décisionnel aux comités de quartier.

Chaque groupe a alors présenté les grandes lignes de son action en faveur de l'air oralement en plénière. Les trois groupes ont été ensuite invités à identifier et à se mettre d'accord de manière exhaustive sur les acteurs et parties prenantes intervenant dans leur projet politique ainsi que les facteurs pouvant avoir un rôle dans la réalisation de l'action (cf. Tableau 3).

Groupe 1 : M. et Mme Velogratos à l'ombre ont deux enfants : Communiquer et Eduquer

-> Forces de l'ordre, médecins, usagers, services communication de la Métropole de Montpellier, TAM, ARS, DREAL, rectorat, universités, services communication des universités, services espaces verts Métropole de Montpellier, SMOOVE.

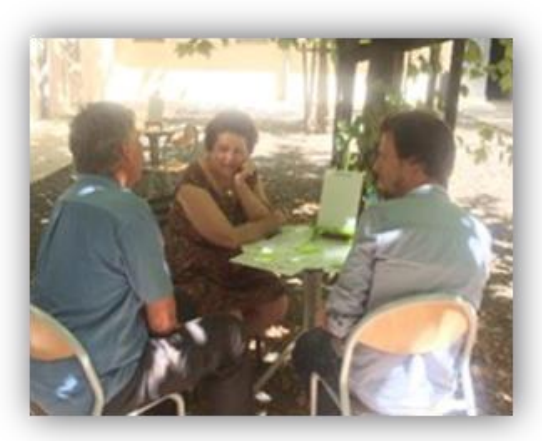

Figure 2. Groupe de famille $n^{\circ} 1$

Groupe 2 : Transition énergétique et aménagement de l'espace

-> Métropole de Montpellier, société civile organisée, grand public, budget participatif, éducation nationale, les établissements scolaires, le conseil régional, l’Europe.

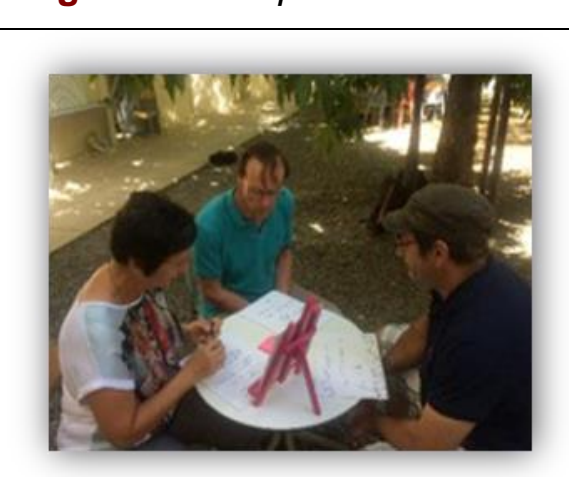

Figure 3. Groupe de famille $n^{\circ} 2$

Groupe 3 : Donner un réel pouvoir décisionnel aux comités de quartier

-> Métropole de Montpellier, calendrier électoral, le Maire de Montpellier (P. Saurel), citoyens, habitants des quartiers, tissu associatif local, proposition de loi, les élus de la Métropole, les comités de quartiers

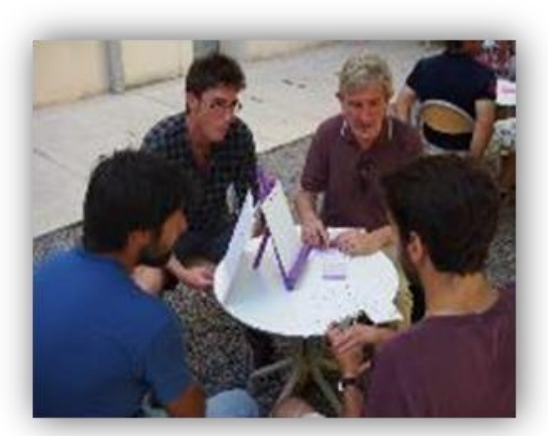

Figure 4. Groupe de famille $n \circ 3$

Tableau 3. Résultats des acteurs clés par famille de mesure sur la qualité de l'air, Montpellier, 03 juillet 2017, @ Sandra Barantal 


\subsubsection{Résultats de l'exercice d'identification des acteurs}

Les familles sont alors invitées à venir mettre sur un support (tableau aimanté) les post-its colorés représentant leurs acteurs et parties prenantes, en respectant leur code couleur (vert, mauve, rose) renvoyant à leur Groupe d'action sur le diagramme arc en ciel. Le choix du positionnement devait avoir fait consensus au sein du groupe d'action avant d'être accroché sur le diagramme. Tous les positionnements sur l'arc en ciel sont débattus. Ainsi, les raisons pour lesquelles un acteur était déposé dans une section ont été discutées par le groupe afin d'en arriver à un consensus.

Les premiers résultats laissent apparaître que les institutions politiques et les usagers sont au cœur des acteurs interagissant avec les différentes actions proposées.

La mesure verte ou Groupe 1 «Monsieur et Madame Velogratos à l'ombre ont deux enfants : communiquer et éduquer » propose donc des actions de mobilité douce dans les zones urbaines et périurbaines en mettant l'accent sur la communication et l'éducation comme leviers de mise en place. Les services techniques (espaces verts notamment) et les usagers du territoire montpelliérain influencent la mesure et sont impactés par les changements qu'elle apporterait dans leur quotidien et leur rythme de travail. Sur le plan de l'éducation, le rectorat et l'Université sont moyennement influents et peu touchés par la mesure pour les mêmes raisons que les acteurs précédents. Les forces de l'ordre identifiées comme des acteurs clés de la mesure $\mathrm{n}^{\circ} 1$, sont les moins touchés par la mesure mais ont toutefois une influence même minime. La TAM (société de Transports de l'Agglomération de Montpellier) est un des acteurs clés qui a une forte influence sur la mesure et son développement, tout autant que le service communication de l'institution politique locale. La DREAL (Direction Régionale de l'Environnement, de l'Aménagement et du Logement) quant à elle a une moindre influence sur la mise en œuvre de la mesure proposée par les participants mais est tout de même citée.

La mesure rose ou encore la mesure du Groupe 2: «Transition énergétique et aménagement » a permis d'identifier un certain nombre d'acteurs clés qui ont été répartis en fonction de leur degré d'influence et d'impact dans sa mise en application sur le territoire montpelliérain. Ainsi, l'acteur clé le plus touché est le grand public, à l'opposé de l'Éducation nationale qui est la moins touchée. Les budgets participatifs des institutions publiques ont un degré d'influence moyen sur la mesure. Le plus influent dans la mise en œuvre est l'institution publique locale, à savoir la Métropole. Le moins influent est l'Éducation nationale qui est suivie de près par la société civile organisée et le Conseil Régional. Enfin, le grand public, identifié comme acteur clé un degré d'influence modéré dans la mise en application de cette mesure.

La mesure mauve du Groupe 3 : «Donner un réel pouvoir décisionnel aux comités de quartier » quant à elle, vise à donner, comme leur appellation, l'indique un réel pouvoir décisionnel aux comités de quartier sur les enjeux de qualité de l'air extérieur. Ils semblent être très influencés dans sa mise en œuvre par la municipalité. Les comités de quartiers existants sont impactés et influents dans la mise en œuvre de cette action. Le tissu associatif est modérément impacté et influent sur la mesure. Les participants ont identifié des facteurs qu'ils estiment clés dans la mise en application de la mesure créée, à savoir, des propositions de lois et les élections politiques locales. Ces agendas ont un degré moyen d'impact et d'influence sur la mesure. Enfin, les citoyens sont modérément touchés par la 
mesure contrairement au Président Maire de Montpellier Méditerranée Métropole qui est très touché par cette mesure qui tend à conditionner son poste de décisionnaire.

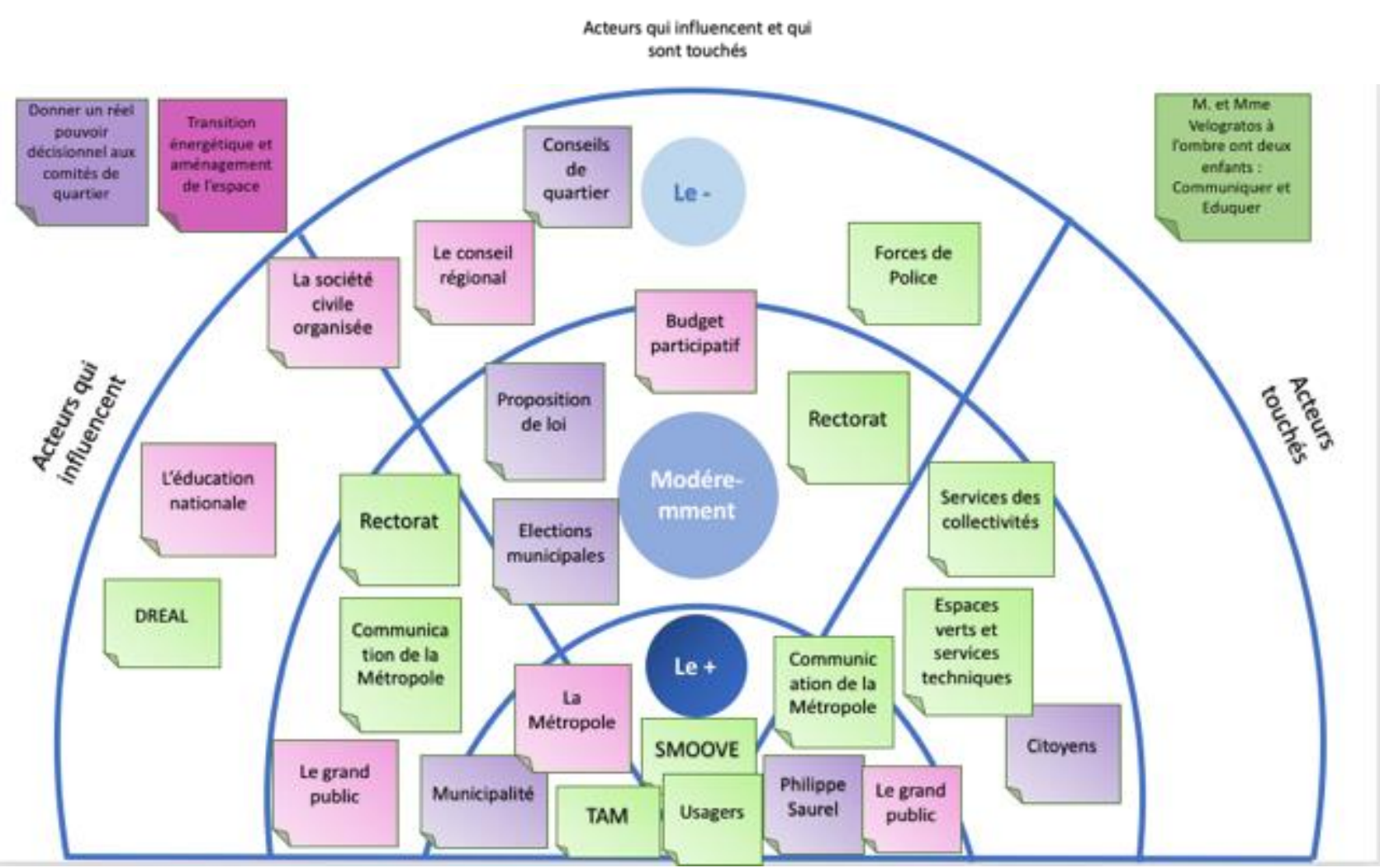

Figure 3. Résultats retranscrits diagramme "analyse des parties prenantes", atelier qualité de l'air, Juillet 2017, Davia Dosias Perla

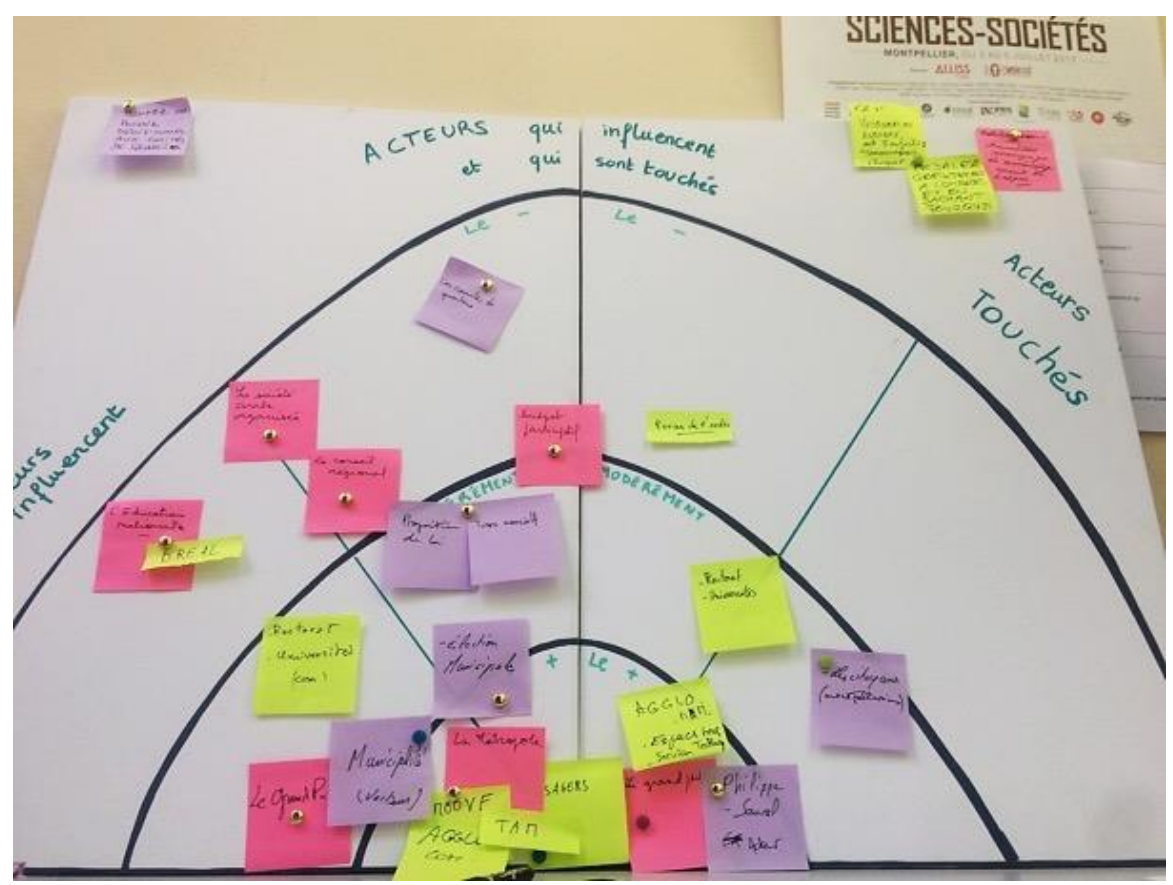

Figure 4. Résultats diagramme " analyse des parties prenantes », atelier qualité de l'air, Juillet 2017, () Sandra Barantal 


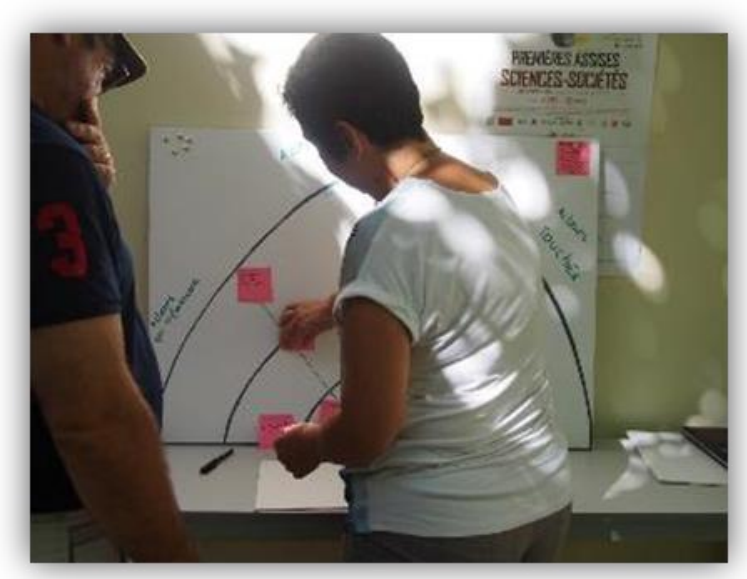

Figure 7. Temps de construction et débat autour des premiers résultats de l'exercice "analyse des parties prenantes ", atelier qualité de l'air, Juillet 2017, (c) Lionel Scotto d'Apollonia

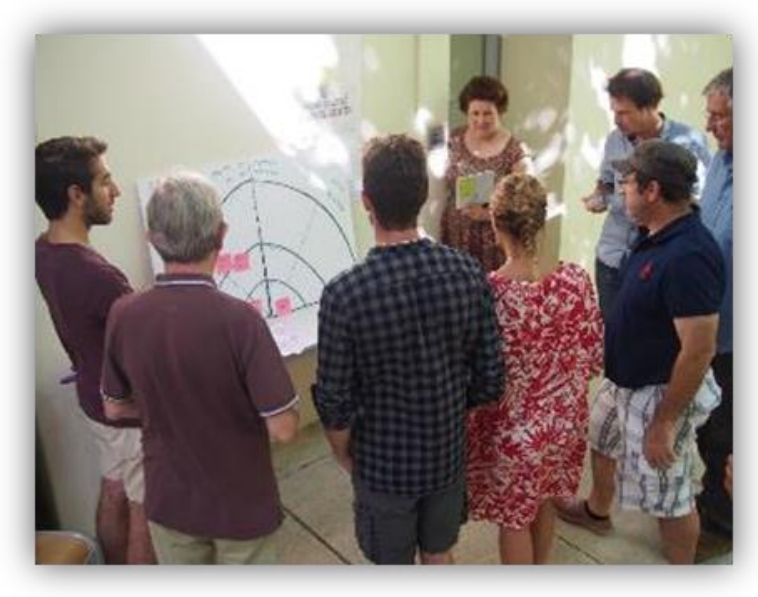

Figure 8. Debriefing autour des résultats co construits de l'exercice " analyse des parties prenantes », atelier qualité de l'air, Juillet 2017, (C) Lionel Scotto d'Apollonia

\subsection{Analyse des résultats et discussion}

\subsubsection{Analyse des résultats}

Cet exercice ne correspond pas encore à un atelier participatif dans le cadre d'une recherche menée sur le territoire ${ }^{27}$ mais il en est la phase antérieure et préparatoire. Il représente un exercice de simulation fort instructif. Ainsi, il minimise les risques lors de son déploiement sur le territoire et contribue à valider son opérativité, ses limites et ses avantages. Ce pré-test, bien que les résultats soient partiels, permet de valider les grandes lignes méthodologiques du design des ateliers à venir.

\subsubsection{Les difficultés techniques à animer un atelier en extérieur}

L'analyse des résultats a été faite à la fin de l'atelier pendant le debriefing, en fin d'exercice d'analyse des parties prenantes. Au regard des conditions extérieures, l'enregistrement audio n'est pas suffisamment de bonne qualité pour saisir l'ensemble des discussions et pouvoir en ce sens reproduire les verbatim. Bien que l'enregistreur soit de très bonne qualité (Zoom H2n), dans le cadre d'un atelier conduit en extérieur, il aurait été préférable d'équiper chaque participant d'un micro « cravate ».

\subsubsection{Validation du design}

Les participants n'ont pas rencontré de difficulté à s'approprier la thématique technique de l'atelier et ont rapidement identifié des actions à mettre en œuvre ou à améliorer pour agir en faveur de la qualité de l'air : développer des actions de transition énergétique en aménageant les quartiers différemment, développer des voies de mobilité douces accessibles à tous en travaillant sur les volets éducatifs et communicationnels ou encore redonner un réel pouvoir décisionnel aux instances existantes de conseils de quartier. Les grandes 
tendances de l'exercice des chaises musicales qui faisaient ressortir massivement l'enjeu sanitaire lié à la qualité de l'air (5 représentations sur 12) ont été reproduites dans les autres exercices, nous retrouvons les enjeux d'énergie, d'aménagement, de mobilité, de bien-être, de bien commun et de décision associés à la qualité de l'air. Ceci est un des éléments renforçant la pertinence du design.

4.2.1.3. Comparaison familles et textes en vigueur à l'échelle de la Métropole montpelliéraine

Les représentations des participants et les actions proposées, peuvent être retrouvées à l'identique dans les grandes politiques publiques en vigueur (Plan Prévention pour l'Atmosphère, Plan de Déplacement Urbain, Politiques de la Ville, Plan Local de l'Urbanisme). Notons que les enjeux relatifs à ladite démocratie participative, qui a été développée par le groupe numéro trois « Donner un réel pouvoir aux conseils de quartier » et qui s'ancre pleinement dans la ligne directrice affichée par l'équipe décisionnelle de la Mairie et Métropole montpelliéraines ne se retrouve dans aucune des politiques publiques prenant en compte les enjeux de qualité de l'air.

Pour illustration partielle voici recoupées dans la tableau 3 les différentes actions issues des plans et politiques publiques (PPA, PCET, PDU, SCoT) en vigueur à l'heure actuelle du territoire de la Métropole de Montpellier et qui prennent en compte les enjeux qualité de l'air, avec les actions issues de l'atelier participatif. 


\begin{tabular}{|c|c|c|c|c|}
\hline Politiques publiques sur r'air & $\begin{array}{l}\text { PCET Métropole } \\
\text { Montpellier } \\
\text { 2013-2018 }\end{array}$ & $\begin{array}{c}\text { SCoT Métropole } \\
\text { Montpelier } \\
\text { Révision en date du } \\
27 \text { avril } 2018 \\
\text { «Document } \\
\text { d'orientation et } \\
\text { d'objectif » } \\
\text { DOCOB }\end{array}$ & $\begin{array}{c}\text { PDU } \\
\text { Montpellier } \\
\text { Agglomération } \\
2010 \text { - 2020 }\end{array}$ & $\begin{array}{c}\text { PPA de l'aire } \\
\text { urbaine de } \\
\text { Montpellier } \\
2018\end{array}$ \\
\hline $\begin{array}{l}\text { Mesure VERTE } \\
\text { « Monsieur et Madame } \\
\text { Velogratos à l'ombre } \\
\text { ont deux enfants : } \\
\text { communiquer et } \\
\text { éduquer» }\end{array}$ & $\begin{array}{l}\text { Renforcer l'attractivité } \\
\text { du réseau de } \\
\text { transports urbains et } \\
\text { des moyens de } \\
\text { transports mixtes et } \\
\text { combinés } \\
\text { Développer l'usage du } \\
\text { vélo à assistance } \\
\text { électrique sur le } \\
\text { territoire }\end{array}$ & $\begin{array}{l}\text { Interconnecter les } \\
\text { réseaux de transports } \\
\text { et les Pôle } \\
\text { d'Échanges } \\
\text { Multimodaux }\end{array}$ & $\begin{array}{l}\text { Augmenter la } \\
\text { part des modes } \\
\text { alternatifs à la } \\
\text { voiture de l'ordre } \\
\text { de plus de } 65 \% \text { à } \\
\text { l'échelle de la } \\
\text { ville de } \\
\text { Montpellier à } \\
\text { l'horizon } 2020 \\
\text { Diminuer les } \\
\text { vitesses pour } \\
\text { passer en zones } \\
\text { de cohabitation } \\
\text { piétonne, } \\
\text { voitures, vélos } \\
\text { Densifier les } \\
\text { maillages vélos }\end{array}$ & $\begin{array}{l}\begin{array}{l}\text { Développer les } \\
\text { mobilités actives }\end{array} \\
\text { Planification de } \\
\text { la mobilité }\end{array}$ \\
\hline $\begin{array}{c}\text { Mesure rose } \\
\text { × Transition énergétique } \\
\text { et aménagement » }\end{array}$ & $\begin{array}{l}\text { Amplifier le recours } \\
\text { aux énergies } \\
\text { renouvelables sur le } \\
\text { territoire }\end{array}$ & $X$ & $X$ & $\begin{array}{l}\text { Elaboration d'un } \\
\text { Schéma } \\
\text { Directeur de } \\
\text { l'Energie } \\
\text { Diminuer les } \\
\text { consommations } \\
\text { d'Energie et } \\
\text { encourager le } \\
\text { développement } \\
\text { des énergies } \\
\text { renouvelables }\end{array}$ \\
\hline $\begin{array}{c}\text { Mesure violette } \\
\text { «Donner un réel } \\
\text { pouvoir décisionnel aux } \\
\text { comités de quartier» }\end{array}$ & $X$ & $X$ & $X$ & $X$ \\
\hline
\end{tabular}


Tableau 4. Analyse comparative des plans de politiques publiques en vigueur sur le territoire Montpelliérain et les propositions d'actions issues de l'atelier

\subsubsection{L'éternelle question de la place du citoyen et de sa capacité d'agir}

L'analyse collaborative des résultats de l'atelier s'est centrée sur deux composantes : 1) le citoyen et 2) la mise en œuvre d'action publique en faveur de la qualité de l'air. Au cours de l'atelier nous avons noté un apparent frémissement de mobilisation citoyenne dans le cadre d'une activité de recherche sur la qualité de l'air. Le terme de frémissement est employé dans le sens où des acteurs de la société dite civile étaient présents dans l'atelier de recherche. Ce point, loin d'être anodin est repris dans la discussion.

\subsubsection{Discussion}

L'analyse des résultats permet de faire ressortir clairement les points positifs à reproduire dans la structuration méthodologique des futurs ateliers et de la «bonne » conduite du projet Air Climat Santé Société \& Art. Ils nous ont aussi permis d'affiner les hypothèses de travail et de mettre au jour les limites d'une telle démarche dont la pertinence ne pourra véritablement s'apprécier qu'à la fin du projet.

D'autre part, elle soulève beaucoup de questions et met au jour les limites d'une telle démarche dont la pertinence ne pourra véritablement s'apprécier qu'à la fin du projet.

\subsubsection{Des jalons pertinents pour la suite du projet grâce à une cartographie des} acteurs/actions pour l'amélioration de la qualité de l'air

Cette première cartographie des acteurs/actions intervenant dans l'amélioration de la qualité de l'air sur le territoire de la Métropole montpelliéraine pourra avantageusement servir de cadre de référence pour les futurs ateliers. Elle permettra en ce sens d'identifier plus aisément les acteurs et les parties prenantes qui seront amenés à constituer les groupes multi acteurs du projet Air Climat Santé Sociétés et Art.

La cartographie élaborée lors de l'atelier participatif met au jour clairement, une régulation top down de l'État et des instantes institutionnelles. En effet, les collectivités territoriales, sur le terrain, en pratique, peinent à prendre en charge pleinement la mise en place cohérente du Plan climat imposé par l'État, traduit en 2010 en Plan Climat Énergie Territoriale (PCET) et précisé en 2016 en Plan Climat Air Énergie Territoriale (PCAET). La nécessité réglementaire de mettre en synergie l'ensemble des acteurs et une concertation citoyenne prenant en compte l'enjeu qualité de l'air, apparait de manière injonctive dans les habitus des collectivités. Cette représentation se retrouve tant au niveau des élus qui tendent depuis plusieurs années à se référer sur ces aspects techniques sur les services (ingénieurs territoriaux, administratifs, agents de terrain) qu'au niveau des services techniques qui travaillent à la mise en œuvre des documents d'objectifs et actions des politiques publiques. Les efforts de l'ADEME pour guider et venir en appui aux collectivités territoriales ne parviennent pas encore à compenser d'une part la délégation au pas de charge et d'autre part l'appropriation de ces enjeux de qualité de l'air par les élus et les agents territoriaux. Les collectivités continuent de déléguer à des bureaux d'études une bonne partie du travail. Le champ de la concertation devient donc un secteur économique à part entière conduit par de potentiels experts ou consultants extérieurs dont les intérêts (soumis à un système concurrentiel important) ne sont pas de facto en cohérence avec les objectifs fixés 
initialement. Nous sommes encore bien loin de l'appropriation institutionnelle de la démocratie participative et des enjeux de qualité de l'air.

\subsubsection{Le paradoxe de la démarche participative}

Les résultats permettent d'esquisser les contours d'un paradoxe entre la volonté de mobiliser le citoyen de façon continue alors que ce dernier ne se mobilise que sur des causes ponctuelles comme par exemple les problèmes inhérents aux sites d'incinérateur de déchets, ou en cas de pic de pollution. Sur le plan de la recherche ce paradoxe pose une question de fond quant à la capacité du dispositif et plus largement de la RAP à pouvoir investir la fabrique participative des politiques publiques. A ce stade, bien que les résultats soient très partiels, il est néanmoins possible de discuter une des limites du dispositif concernant sa propre «posture» par rapport à la mobilisation. En effet, l'analyse fait ressortir un frémissement de la participation citoyenne. La portée de l'exercice présenté ici est donc très limitée, voire problématique dans le sens où l'atelier n'a pas permis de générer une mobilisation massive, de prendre en compte les aspects conflictuels ni ne vient pas en réponse à une mobilisation citoyenne. L'analyse des résultats fait apparaitre clairement un diagnostic très consensuel, le « citoyen lambda » rencontré la veille dans la rue étant absent. Or il existe de nombreuses problématiques autour de Montpellier, chaque incinérateur étant une source de conflits sociaux et de battages médiatiques influençant plus ou moins les orientations et les discours politiques. Il peut donc apparaître paradoxal de mobiliser en l'absence d'une volonté de mobilisation. Malgré toutes les limites inhérentes à cet atelier, il apparait ici l'hypothèse que l'échelle d'une Métropole soit encore une dimension trop importante et qu'il faille réduire davantage le maillage à des zones plus restreintes en faisant ainsi écho aux usages des habitants dans leur espace de vie et en faisant appel à leur rapport sensible à leur territoire.

L'atelier a permis de mettre au jour un autre paradoxe entre les volontés (réglementaires) d'implication du citoyen des pouvoirs publics et son manque d'implication réelle dans les processus de construction de la décision publique. A titre d'exemple les citoyens ont été invités à participer à proposer des actions en faveur de la qualité de l'air par un lien internet sur le site de la DREAL LR mais n'ont jamais été conviés aux réunions de Comité de Pilotage pour la reformulation de la feuille de route du Plan Prévention de l'aire urbaine de Montpellier qui devait proposée des actions concrètes et socialement adaptée dans un pas de temps relativement court (opérationnalité avant la fin du quinquennat). Dans le cadre de l'atelier et relavant quasiment d'un exercice tautologique, la discussion des participants s'est centrée sur le fait que le citoyen apparaisse comme un des acteurs permettant une mise en place cohérente et efficace des politiques publiques avec une influence sur l'action relative.

Les premiers résultats présentés dans cet article concernant le déficit de mobilisation peuvent se retrouver dans d'autres études. Par exemple, les spécialistes des alertes et des risques sanitaires, Francis Chateauraynaud et Josquin Debaz ont analysé ce déficit de mobilisation citoyenne concernant la pollution aux particules fines dont les conséquences sont connues et médiatisées dans l'espace public (asthme, maladies cardio-vasculaires, cancers) alors que d'autres enjeux aux conséquences bien plus controversées mobilisent un très large public [CHAT 12]. L'existence de ce profond paradoxe est d'autant plus socialement significatif que l'expérience sensible de la pollution de l'air est partagée de tous et que les alertes sur les pollutions atmosphériques, pollutions les plus anciennes dans l'histoire environnementale [CHAR 15] [MAS 10] sont portées et traitées par des organismes et instances officielles, tout niveau d'échelle confondu notamment par les 
moyens d'informations de mesures journalières comme nous pouvons trouver sur le site par exemple d'ATMO OCCITANIE ${ }^{28}$. Normées par un cadre réglementaire, sont en effet prévus dans les Plans Climat Air Énergie Territoriaux, des orientations et des campagnes d'information (notamment dans le mandat d'ATMO OCCITANIE) et de "sensibilisation » des acteurs: les élus, les responsables de services et leurs équipes, les habitants et l'ensemble des acteurs du territoire.

De plus, les mobilisations citoyennes qui s'agrègent autour de causes ponctuelles, en général s'accompagnent d'une métrologie citoyenne spécifique, qui d'ailleurs était la grande absente des débats de l'atelier participatif. Le problème de cette métrologie réside principalement dans le fait qu'elle ne répond pas aux normes en vigueur et cristallise les tensions [CHAT 13]. En ce sens le dispositif Artivistes-atelier développe sur le même territoire du Pays de l'Or, une recherche avec l'UMR Géosciences de l'Université de Montpellier qui vise à développer un programme de sciences citoyennes autour d'un processus de bio détection des particules fines via les plantes du territoire. Ce programme fait suite à une mobilisation citoyenne générée par le doublement de l'autoroute A9 qui a donné lieu à la mise en place de cette métrologie citoyenne expérimentale (sur la base d'un banc expérimental spécialement dédié et d'une analyse géochimique isotopique) doublée de capteur standard. La force de ce projet, en phase initiale lui aussi, est de permettre de créer des cartographies dynamiques des polluants atmosphériques répondant aux normes en vigueur et surtout caractère innovant la détermination de leur origine. Ces cartographies dynamiques issues d'un programme de métrologie citoyenne visent à alimenter en retour le projet d'accompagnement des politiques publiques.

\section{Conclusion}

La qualité de l'air que nous respirons, un sujet qui mobilise peu ou trop : le dispositif au cœur de la mobilisation des acteurs

Les résultats présentés dans cet article reposent sur un «petit» atelier conduit dans un contexte particulier. Compte-tenu des nombreuses limites discutées précédemment, les résultats et leurs limites permettent de nourrir la réflexion sur la mobilisation concernant la qualité de l'air. En effet les limites du dispositif, qui se retrouve en quelque sorte au milieu du gué, c'est-à-dire en tension entre un déficit ou un excès de mobilisation, sont assez révélatrices de la réalité sociale concernant l'amélioration de la qualité de l'air.

A ce stade, la question d'une participation citoyenne dans la fabrique des politiques publiques sur la qualité de l'air représente un vaste champ exploratoire pour la recherche. Faisant pleinement écho à cette situation, la cartographie dynamique des actants clés (acteurs et institutions) et des familles d'actions co-construite dans le cadre de l'atelier participatif décrit dans cet article est un des outils permettant d'établir ce qu'il est commun d'appeler une vision partagée et transversale du territoire et de ses enjeux notamment sur la lutte contre la pollution de l'air. Il reste à apprécier sa portée dans le projet Air Climat Santé Société \& Art.

Un frein dans la mise en œuvre d'une politique publique sur l'air: le manque d'articulation de la transversalité

${ }^{28}$ ATMO Occitanie est l'observatoire de la qualité de l'air en Occitanie. Consulté en ligne le 5 mai 2018 sur le lien d'information sur : http://atmo-occitanie.org/. 
Désormais à l'échelle plus locale des collectivités territoriales, l'enjeu relatif à la qualité de l'air dans les Plans Climat Air Énergie Territoriaux (PCAET) est couplé à celui de l'adaptation des territoires et des usages au changement climatique. Ce surplus de complexité n'est pas un facteur de facilitation des politiques publiques sur la qualité de l'air, ses enjeux spécifiques pouvant rapidement être gommés par la structuration en silo des collectivités territoriales. La construction des PCAET s'articule avec les documents de planification et d'orientation du territoire plus larges tel que les Schémas de Cohérence Territoriale et de fait avec un plus large panel d'acteurs du territoire pour établir le diagnostic de départ.

L'analyse des premiers résultats de l'atelier participatif sur l'air permet d'affiner une hypothèse de travail relative à la difficile voire à l'inexistante traduction effective des outils et des moyens de la démocratie participative dans ces politiques publiques prenant en compte l'enjeu de la qualité de l'air dans le PCAET, tout autant que les SCoT, PDU, PPA. L'hypothèse de travail renforce donc la pertinence de questionner cette articulation afin de permettre de la renforcer. En ce sens, le dispositif révèle un potentiel heuristique fécond dans le sens où il permet d'interroger in vivo la transition d'une politique sectorialisée sur l'air faisant appel à plus de transversalité.

En outre, ces résultats renforcent une hypothèse initiale et permet d'orienter plus finement un axe de recherche visant à interroger ce que produit une potentialisation de la transversalité dans la prise en charge des enjeux socioenvironenmentaux par les services techniques des collectivités. En général la transversalité est quasi inexistante ou par défaut portée à l'initiative d'une personne dont l'exercice dans le temps reste limité. En ce sens, il apparaît pertinent d'observer et d'analyser ce qu'une potentialisation de la transversalité génère comme effet dans la traduction des directives réglementaires en actions publiques.

Reste à déterminer ce qu'une transversalité plus effective produit sur la participation citoyenne cantonnée à l'heure actuelle au cadre réglementaire d'une simple concertation. Dans cette optique, le dispositif doit exercer sa volonté à être critique et réflexif autant que faire se peut tout en engageant l'ensemble des acteurs dans cette voie. En évitant les écueils d'une approche trop «naturalisante » du social, le dispositif Artivistes-atelier revient à produire un artefact dans la fabrique participative des politiques publiques sur l'air. La réponse induite par cet artefact permet avec toutes les réserves nécessaires de produire des données de recherche dont la pertinence doit être soumise à un examen collectif. Cette posture de recherche est une façon originale et expérimentale d'analyser aux échelles locales, les modalités de régulation des politiques publiques sur la qualité de l'air. Dans la logique de la RAP, l'objectif vise, à supposer que des réformes soient possibles effectivement, une amélioration des problèmes liés à la sectorisation des politiques publiques environnementales dichotomisées dans une pluralité de services techniques spécialisés au sein des collectivités. Le dispositif par son ancrage dans la recherche et dans l'expertise doit permettre de prendre en compte les systèmes de gouvernance et les éventuelles tensions internes, facteurs limitant la lisibilité et la mise en cohérence d'actions sur un territoire.

Le design présenté n'est pas réservé exclusivement aux enjeux locaux du territoire Montpelliérain et peut avantageusement s'adapter aux spécificités d'autres territoires. L'actuelle formulation et mise en ouvre des différents PCAET à l'échelle de la France constitue un laboratoire à ciel ouvert de la fabrique dite participative des politiques 
publiques sur les enjeux de qualité de l'air. La fabrique participative des politiques publiques sur l'air restera-t-elle l'arlésienne?

\section{Remerciements}

Nous tenons à remercier pour leur soutien : le Labex Cemeb et le Labex DRIIHM, l'OHMI NUNAVIK et la MSH Sud, l'UMR ART Dev, ainsi que l'ensemble des participants et partenaires autour du dispositif Artivistes-atelier et du GDR PARCS.

\section{Bibliographie}

[BAU 10] BAUTES, N., «L'expérience artiviste dans une favela de Rio de Janeiro », Cahiers de géographie du Québec, $\mathrm{n}^{\circ} 153$, vol.54., p. 471-498, déc-jan, 2010-2011.

[BAR 11] BARBIER, R., LARRUE, C. «Démocratie environnementale et territoires: un bilan d'étape. Participations ». De Boeck Supérieur. Vol 1, n $1,2011$.

[BEC 01 (86)] BECK, U. La société du risque. Sur la voie de la modernité, trad. de l'allemand par L. Bernardi. Paris. Aubier, 521p, 2001 [1986].

[BEV 02] Bevort, A. Pour une démocratie participative. Paris : Presses de Sciences Po (P.F.N.S.P), 2002.

[BLA 17] Blangy, S. Co-construire le tourisme autochtone par la recherche action participative et les technologies de l'information. Une nouvelle approche de la gestion des ressources et des territoires. L'HARMATTAN. ISBN: 978-2-343-05506-0- 664 pages, 2017.

[BLO 08 a] BlONDIAUX, L. Le nouvel esprit de la démocratie. Actualité de la démocratie participative, Le Seuil, Paris coll. La république des idées, 2008.

[BLO 08 b] BlondiauX, L. « Démocratie délibérative vs. Démocratie agonistique ? Le statut du conflit dans les théories et les pratiques de participation contemporaine », Raisons politiques, Vol 2 (30), p. 131-147, 2008.

[BLO 11] BlONDiAuX L., FouRniau J.-M. «Un bilan des recherches sur la participation du public en démocratie: beaucoup de bruit pour rien ? », Participations, Vol 1 (1), p. 8-35, 2011.

[BOE 12] BOEUF, G., et al. «L'apport des sciences participatives à la connaissance de la biodiversité en France », La lettre de l'OCIM, 144, pp8-18, 2012.

[BOL 91] BoltAnski, L. et ThÉVEnot, L. De la justification. Les économies de la grandeur. Gallimard, Paris, 1991.

[BOU 97] BOURDIEU, P. Méditations pascaliennes, Seuil, Paris, 1997.

[BOU 01] BOURDIEU, P. Science de la science et réflexivité, Raisons d'agir, Paris, 2001.

BuClet, N. et SALOMON, D. «Les processus participatifs transforment-ils les représentations des élus ruraux sur leur rôle et leur territoire ? » Espaces et sociétés, Paris, Erès, pp123-138, 2012.

[BOY 15] BoY, D. «Les représentations sociales du changement climatique ». Pollution Atmosphérique, Numéro spécial «Adaptation au changement climatique et maîtrise de la qualité de l'air », p. 190-201, 2013.

[CAL 09] CAllon, M., LAscoumes, P., BARThe, Y. Agir dans un monde incertain. Essai sur la démocratie technique, Le Seuil, Paris, 2001.

[CEF 07] CEFAÏ, D. Pourquoi se mobilise-t-on? Les théories de l'action collective, La Découverte, Paris, 2007.

[CHAR 15] CHARVOLIN, F., et al. Un air familier - Socio histoire des pollutions atmosphériques, Les Mines - Paristech, 2015. 
[CHAT 12]. Chateauraynaud, F., Debaz, J. « Des alertes à couper le souffle. Point sociologique sur les particules fines et la pollution atmosphérique », Socio-informatique et et argumentation, 15 octobre 2012. Article en ligne consultable le 6 mai 2018 sur le lien suivant : https://socioargu.hypotheses.org/4129.

[CHAT 13]. Chateauraynaud, F., Debaz, J. « De la métrologie en démocratie. La nouvelle vague des capteurs citoyens », Socio-informatique et argumentation, 30 septembre 2013. Article en ligne consultable le 6 mai 2018 sur le lien suivant : https://socioargu.hypotheses.org/4505\#identifier_17_4505.

[CHAT 17] Chateauraynaud, F., Debaz, J., Aux bords de l'irréversible. Sociologie pragmatique des transformations, Paris: Petra, 2017.

[CHEVA 11] Chevalier, J-M., Bourassa, M., BuCKLES, D-J. Guide de la recherche action, la planification et l'évaluation participatives en éducation, Gatineau, SAS2 Dialogue, 2011.

[CHEVI 03] Chevigné, S. «Expertise, risque et implication des profanes : autour de la conférence de citoyens à propos des OGM », dans GILBERT. C. Risques collectifs et situations de crise. Apports de la recherche en sciences humaines et sociales, L'Harmattan. p. 267-278, 2003.

[COM 15 a] COMBY, J-B. «La politisation en trompe-l'œil du cadrage médiatique des enjeux climatiques après 2007 », Le Temps des médias, vol. 25, no. 2, 2015, pp. 214-228, 2015.

[COM 15 b] COMBY, J-B. La question climatique, Genèse et dépolitisation d'un problème public. Paris : Raisons d'agir, 2015.

[DES 07] Desjardins, X., LerouX, B. «Les schémas de cohérence territoriale : des recettes du développement durable au bricolage territorial », Flux 3/2007, n 69, 2007.

[DUB 09] DUBOIS, J. Les politiques publiques territoriales: La gouvernance multi-niveaux face aux défis de l'aménagement, PUR, 2009.

[FOU 07] FOURNIAU, J-M. «L'expérience démocratique des "citoyens en tant que riverains" dans les conflits d'aménagement », Revue européenne des sciences sociales, XLV (1), p. 149-179, 2007.

[FOU 11] FOURNIAU, J-M. «L'institutionnalisation controversée d'un modèle français de débat public », Télescope, 17 (1), p. 70-93, 2011.

[GON 14] GONZALES-LAPORTE, C. Recherche-action participative, collaborative, intervention... Quelles explications? [Rapport de recherche] Labex ITEM. 2014.

[HAB 87; 97] HABERMAS, J. Théorie de l'agir communicationnel. Trad. fr. 1987, rééd 2001 pour le t.I, Payard pour le t.II. 1987, 1997.

[IPP 96] IPPC, Directive IPCC (Integrate Pollution Prevention and Control), 1996.

[JOD 89] JODELET, D. Les représentations sociales, PUF, Paris, 1989.

JOUZEL, J. L'Atelier Climatique Citoyen, http://artivistes.neowordpress.fr/atelier-climatique-citoyen, lien consulté le 25/02/2018, 2016

[JOUZ 14] JouZel, J., Debroise, A. Le défi climatique. Objectif : $2^{\circ}$ C !, Quai des Sciences, Dunod, 2014.

[LE GA 95] LE GALÈS, P. «Les réseaux d'action publique entre outil-passe partout et théorie de moyenne portée », dans LE GALÈs, P., THATCHER, M. (dir.), Les réseaux de politique publique. Débat autour des policy networks, L'Harmattan, Paris, 1995.

[LE M 10] LE MAREC, J. Les études de sciences: pour une réflexivité institutionnelle. L'Harmattan, Paris, 2010 .

[MAB 17] MABI, C. «Citoyen hackeur. Enjeux politiques des civic tech ». La vie des idées, 2017.

[MAS 10] Massard-Guilbaud, G. Histoire de la pollution industrielle: France, 1789-1914, Paris, Éditions de l'EHESS, 2010

[MAY 05] MAYERS, T. (Re) Writing Craft : composition, creative writing and the futur of English, UPP. p.2, 2005. 
[MER 05] MERMET, L. et al. «L'analyse de la gestion environnementale: un cadre théorique pour penser l'efficacité en matière d'environnement », Natures Sciences Sociétés, Vol. 13, no. 2, 2005, pp. 127-137, 2005.

[MON 92] MONIER, E., Duran, P. « Le développement de l'évaluation en France. Nécessités techniques et exigences politiques », Revue française de science politique, pp235-262, 1992.

[MOS 61] MoscovicI, S. «La psychanalyse, son image et son public », Revue française de sociologie, pp328-330, 1961.

[PÉC 05] PÉCAUD, D. « Risques et précautions : l’interminable rationalisation du social », La Dispute, 2005.

[PER 06] PÉRetTi-WATel, P., HAMmer, B. «Les représentations profanes de l'effet de serre », Natures Sciences Sociétés, 14(4), p. 353-364, 2006.

[PIT 10] PITSEYS, J. « Le concept de gouvernance», Revue interdisciplinaire d'études juridiques 2010/2 (Volume 65), p. 207-228. (2010).

[SAL 06] SALLES, D. Les défis de l'environnement. Démocratie et efficacité. Paris, Syllepses, 2006.

[SAL 13] SAlles, D., LEROY, P. «Gouvernance environnementale », in CASTILLO, (I.) avec BARBIER (R.), BLONDIAUX (L.), CHATEAURAYNAUD, (F.), FOURNIAU (J.-M.), NEVEU, (C.) et SALLES, (D.). Dictionnaire critique et interdisciplinaire de la participation (2013). Consulté en ligne le 25 avril 2017 sur le lien suivant : http://www.dicopart.fr.

[SCO 17] SCOTto D'APOllonia, L., Dosias-Perla, D. «L'expérience Artivistes-atelier, l'illusion participative», dans GIS DEMOCRATIE ET PARTICIPATION, Les expérimentations démocratiques d'aujourd'hui : convergences, fragmentations, portées politiques, Paris, janvier 2017

[THE 02] THEYS, J. L'approche territoriale du « développement durable », condition d'une prise en compte de sa dimension sociale, Développement durable et territoires [en ligne], Dossier 1, mis en ligne le 23 septembre 2002. URL: http://developpementdurable.revues.org/1475 ; DOI : 10.4000/developpementdurable.1475, 2002/

[THE 06] TheVEnOt, L. L'action au pluriel. Sociologie des régimes d'engagement. La Découverte, Paris, 2006.

[ZEL 14] ZELEM, M.-C., BESLAY, C. La sociologie de l'énergie. Gouvernance de l'action publique, pratiques sociales et outils du changement, CNRS Editions, Paris, 2015.

[ZIT 09] ZITOUN, P. Des indicateurs pour gouverner: boussoles ou miroirs déformants, collection « recherche » du PUCA, n 196 , Paris, 2009. 